\title{
The MAVERIC Survey: Still No Evidence for Accreting Intermediate-mass Black Holes in Globular Clusters
}

\author{
Evangelia Tremou ${ }^{1}$ (iD, Jay Strader ${ }^{1}$ (i), Laura Chomiuk ${ }^{1}$ (iD), Laura Shishkovsky ${ }^{1}$, Thomas J. Maccarone ${ }^{2}$, \\ James C. A. Miller-Jones ${ }^{3}(1)$, Vlad Tudor $^{3}$, Craig O. Heinke ${ }^{4}$, , Gregory R. Sivakoff $^{4}$ (i), Anil C. Seth ${ }^{5}$, and Eva Noyola ${ }^{6}$ \\ ${ }^{1}$ Center for Data Intensive and Time Domain Astronomy, Department of Physics and Astronomy, Michigan State University, \\ East Lansing, MI 48824, USA; tremou@msu.edu \\ ${ }^{2}$ Department of Physics and Astronomy, Texas Tech University, Box 41051, Lubbock, TX 79409-1051, USA \\ ${ }^{3}$ International Centre for Radio Astronomy Research, Curtin University, GPO Box U1987, Perth, WA 6845, Australia \\ ${ }^{4}$ Department of Physics, University of Alberta, CCIS 4-181, Edmonton, AB T6G 2E1, Canada \\ Department of Physics and Astronomy, University of Utah, Salt Lake City, UT, USA \\ ${ }^{6}$ McDonald Observatory, University of Texas at Austin, Austin, TX 78712, USA \\ Received 2018 February 28; revised 2018 May 11; accepted 2018 May 30; published 2018 July 18
}

\begin{abstract}
We present the results of an ultradeep, comprehensive radio continuum survey for the accretion signatures of intermediate-mass black holes (IMBHs) in globular clusters (GCs). The sample, imaged with the Karl G. Jansky Very Large Array and the Australia Telescope Compact Array, comprises 50 Galactic GCs. No compelling evidence for an IMBH is found in any cluster in our sample. In order to achieve the highest sensitivity to low-level emission, we also present the results of an overall stack of our sample as well as various subsamples, also finding nondetections. These results strengthen the idea that IMBHs with masses $\gtrsim 1000 M_{\odot}$ are rare or absent in GCs.
\end{abstract}

Key words: black hole physics - globular clusters: general - radio continuum: general

\section{Introduction}

Intermediate-mass black holes (IMBHs) have been proposed as a population of black holes (BHs) with masses between those of supermassive BHs that reside at the centers of galaxies (typically $\gtrsim 10^{6} M_{\odot}$ ) and those of stellar-mass $\mathrm{BHs}$ created through the deaths of massive stars $\left(\lesssim 100 M_{\odot}\right)$. IMBHs $\left(10^{2}-10^{5} M_{\odot}\right.$; e.g., Noyola \& Gebhardt 2006 ; Feng \& Soria 2011) draw ongoing interest as promising seeds for the growth of supermassive BHs (Volonteri \& Perna 2005). Many possible origins for IMBHs have been suggested, including the collapse of metal-free Population III stars (Madau \& Rees 2001) or direct collapse from gas in low-mass halos (Loeb \& Rasio 1994; Eisenstein \& Loeb 1995; Bromm \& Loeb 2003; Lodato $\&$ Natarajan 2006). Massive young star clusters have also been suggested as important IMBH formation sites, perhaps via runaway stellar mergers (Portegies Zwart \& McMillan 2002; Gürkan et al. 2004; Portegies Zwart et al. 2004; Vanbeveren et al. 2009). Vesperini et al. (2010) suggested that a central BH can grow through the accretion of gas lost in these star clusters. Another potential channel for forming IMBHs is via sequential mergers of stellar-mass BHs in globular clusters (GCs; Miller \& Hamilton 2002). These possibilities make GCs primary targets for the search for IMBHs.

Efforts to investigate the presence of IMBHs in GCs, and subsequently constrain their masses, utilize two main approaches: dynamical or accretion signatures. Stars residing near the cluster center, inside the putative sphere of influence of any $\mathrm{IMBH}$, have been studied using kinematic measurements such as radial velocities and proper motions (Newell et al. 1976; Gebhardt et al. 2005; McLaughlin et al. 2006; Anderson \& van der Marel 2010; Noyola 2010; Kamann et al. 2014). Compared to dynamical measurements of supermassive BHs in galaxy centers, these IMBH studies are much more challenging owing to the relatively low $\mathrm{BH}$ masses: there are few stars within the IMBH sphere of influence and, especially for lowmass IMBHs, the signature of a central point mass can be confused with a concentrated population of stellar remnants (den Brok et al. 2014). A more recent approach is to constrain the potential using precise timing of one or more radio pulsars (Freire et al. 2017; Kızıltan et al. 2017; Perera et al. 2017). Overall, despite claims of dynamical evidence for IMBHs in many individual Galactic GCs (e.g., Ibata et al. 2009; Noyola 2010; Lützgendorf et al. 2011; Feldmeier et al. 2013; Lützgendorf et al. 2013a), there is no consensus about the presence of an IMBH in any particular object.

An alternative approach, again drawing a parallel to studies of supermassive BHs, is to search for accretion evidence for IMBHs. The source HLX-1, located in the halo of galaxy ESO 243-49 at distance of $95 \mathrm{Mpc}$, offers the best current such case. $\mathrm{X}$-ray, optical, and radio data suggest the likely presence of a $\sim 10^{4}-10^{5} M_{\odot}$ IMBH accreting at close to the Eddington rate (Webb et al. 2012), possibly located at the center of a tidally stripped young nuclear star cluster (Farrell et al. 2014; Musaeva et al. 2015; Soria et al. 2017; Webb et al. 2017).

By contrast, any so-far undiscovered IMBHs in Galactic clusters would necessarily be in quiescence, accreting at an extremely low rate, akin to $\mathrm{Sgr} \mathrm{A}^{*}$. They would therefore be best observed as radio continuum sources due to synchrotron emission from relativistic jets (Maccarone 2004; Maccarone et al. 2005; Maccarone \& Servillat 2008), though in a few nearby clusters with very deep X-ray data these observations can offer comparable or better constraints on accretion than radio data (e.g., Haggard et al. 2013).

The radio methodology has been applied to deep radio imaging of the Galactic GCs M15, M19, and M22 by Strader et al. (2012) to set $3 \sigma$ upper limits of $<1000 M_{\odot}$ on the masses of IMBHs in these clusters and has been extended by Wrobel et al. $(2015,2016)$ to extragalactic GCs in M81 and NGC 1023 (where nondetections were also found, at higher mass limits than for Galactic clusters) using a stacking analysis.

In this paper, we use deep radio observations from the Karl G. Jansky Very Large Array (VLA) and the Australia 
Telescope Compact Array (ATCA) to search for accretion signatures of IMBHs in 50 Galactic GCs, using data obtained as part of the MAVERIC (Milky Way ATCA and VLA Exploration of Radio-sources in Clusters) survey. Our paper is organized as follows: in Section 2, we present the sample of GCs and the radio observations. Section 3 describes our methodology for determining IMBH mass constraints. In Section 4, we present the results for each cluster and the stack of all 50 Milky Way GCs. In Section 5 we discuss and analyze these results. An Appendix considers in detail the radio data for NGC 6624, which has a number of bright known radio sources near its center and a recent IMBH claim (Perera et al. 2017).

\section{Radio Data and Reduction}

\subsection{Target Selection}

The MAVERIC sample was chosen primarily by distance and mass and was intended to be complete for GCs with masses $>10^{5} M_{\odot}$ and distances $<9 \mathrm{kpc}$, keeping in mind that many GCs near the bulge have uncertain distances. Massive clusters $\left(\gtrsim 5 \times 10^{5} M_{\odot}\right)$ at larger distances were added to search for IMBHs. Objects at declinations $\delta>-35^{\circ}$ were primarily observed with the VLA and more southerly sources were observed with ATCA, though this division is not precise and a few clusters were observed with both arrays.

Here, we use the most recent updated distance measurements to sample GCs; distances and references are listed in Tables 1 and 2.

More details on the sample selection, observing setup, and source catalogs will follow in separate survey papers (L. Shishkovsky et al., in preparation; V. Tudor et al., in preparation).

\subsection{VLA}

VLA observations took place during 2011 May-August (NRAO/VLA Program IDs 10C-109 and 11A-269), 2012 September (12B-073) 2014 February-June (13B-014), and 2015 May-August (15A-100 and 15A-225). Data for $18 \mathrm{GCs}$ were obtained in the most extended A configuration, with 7 more southerly clusters observed in the BnA configuration. The remainder of the GCs were observed during "move time" in or out of A configuration. With these extended configurations, we obtained angular resolutions $\lesssim 1^{\prime \prime}$ for nearly all VLA GCs, which facilitates the comparison between radio images and the optical centers. The median synthesized beam in the $5 \mathrm{GHz}$ VLA image (and in the averaged $6 \mathrm{GHz}$ images) is 0 ." $7 \times 0$." 4 .

VLA observations were made with $C$ band receivers (4-8 $\mathrm{GHz}$ ). Data taken from 2011 to 2014 used 8 bit mode, with two independent $1024 \mathrm{MHz}$-wide basebands centered at 5.0 and 6.75 GHz. In 2015, we used 3 bit mode, with two independent $2048 \mathrm{MHz}$-wide basebands centered at 5.0 and 7.0 GHz. The amount of usable continuum for the 3 bit observations was less than double that in the 8 bit observations owing to significant radio frequency interference (RFI) in the band. In both modes the bandwidth was divided into $128 \mathrm{MHz}$-wide spectral windows, and each spectral window was sampled by 64 channels. All observations were obtained in full polarization mode.

We were approved to obtain $10 \mathrm{hr}$ of observations per VLA cluster, which nominally would result in $7-8 \mathrm{hr}$ on source, depending on the length of the individual observing blocks.
The median on-source observing time was consistent with this goal, at $7.4 \mathrm{hr}$. Not all the requested blocks were successfully observed, and three clusters had final on-source times that were less than $5 \mathrm{hr}$ (M54, Liller 1, and NGC 6522), with correspondingly higher rms noise levels. Observations typically alternated between 10 minutes on source and short observations of a phase calibrator (selected to be within 10 degrees of the science target). A bandpass and flux calibrator were observed at the start or end of each block.

Standard procedures were followed for data flagging, calibration, and imaging with the Common Astronomy Software Application package (CASA; McMullin et al. 2007) and the Astronomical Image Processing System (AIPS; Greisen 2003). Imaging of the data was carried out using a Briggs weighting scheme (robust $=1$ ). To mitigate artifacts from large fractional bandwidth, the $u v$ data were divided into two frequency chunks (basebands) and imaged separately. In CASA, frequency-dependent clean components (with two Taylor terms; nterms $=2$ ) were also used in imaging to mitigate large-bandwidth effects (Rau 2012). For the GCs with bright sources that generated considerable artifacts, we applied self-calibration to optimize the image quality.

Of the 29 GCs with VLA data, 17 were taken primarily or only in 3 bit mode, which had $3-3.4 \mathrm{GHz}$ of bandwidth remaining after RFI flagging. The median rms noise in each of the baseband images centered at 5.0 and $7.0 \mathrm{GHz}$ was $2.0 \mu \mathrm{Jy} \mathrm{bm}{ }^{-1}$. For nine GCs observed earlier in the project, the data were taken in 8 bit mode, which typically results in a $1.8 \mathrm{GHz}$ bandwidth after flagging. Given the lower bandwidth, the baseband rms noise values were slightly higher, $2.5 \mu \mathrm{Jy}$ $\mathrm{bm}^{-1}(5.0 \mathrm{GHz})$ and $2.1 \mu \mathrm{Jy} \mathrm{bm}^{-1}(6.75 \mathrm{GHz})$. The three GCs with comparable exposure times in 3 bit and 8 bit mode had noise levels intermediate between the 3 bit and 8 bit median values, as expected.

Given the expected flat spectrum of the radio continuum emission from low-luminosity accreting IMBHs, we also combined the individual basebands into a single image to maximize sensitivity. To do this, the higher-frequency image was convolved to the resolution of the lower-frequency image using the AIPS task CONVL. The high- and low-frequency images were then averaged using the AIPS task COMB, weighting by the variance. The median rms noise at $6.0 \mathrm{GHz}$ is $1.5 \mu \mathrm{Jy} \mathrm{bm}^{-1}$ ( 3 bit data) and $1.7 \mu \mathrm{Jy} \mathrm{bm}^{-1}$ ( 8 bit data). The distribution of the observed image noise in the frequency averaged images is presented for all GCs in Figure 1.

\subsection{ATCA}

27 GCs were observed with ATCA (project code: C2877) in the extended $6 \mathrm{~A}$ or $6 \mathrm{D}$ configurations in runs from 2013 to 2015. Archival data for 47 Tuc and $\omega$ Cen from Lu \& Kong (2011) are also included in our analysis, imaged together with our newer data for these clusters. All observations were carried out using the Compact Array Broadband Backend (Wilson et al. 2011), allowing simultaneous observations in two bands centered at 5.5 and $9.0 \mathrm{GHz}$, each with $2 \mathrm{GHz}$ of bandwidth. Observations were typically made over two to three separate blocks on different days. In each epoch, the target cluster and phase calibrator were alternately observed for 10-20 minutes and 1.5-2 minute integration times respectively, depending on atmospheric stability and calibrator brightness.

Flagging and calibration were performed in MIRIAD (Sault et al. 1995), and imaging was performed in CASA. As with the 
Table 1

\begin{tabular}{|c|c|c|c|c|c|c|c|c|c|c|c|c|c|}
\hline ID & $\begin{array}{l}\text { R.A. (J2000) } \\
\text { (h:m:s) }\end{array}$ & $\begin{array}{l}\text { Decl. (J2000) } \\
\left({ }^{\circ},{ }^{\prime} \prime \prime\right)\end{array}$ & $\begin{array}{l}\text { Uncertainty } \\
\text { (") }\end{array}$ & $\begin{array}{l}\text { Position } \\
\text { Reference }\end{array}$ & $\begin{array}{l}\text { Distance } \\
(\mathrm{kpc})\end{array}$ & $\begin{array}{l}\text { Distance } \\
\text { Reference }\end{array}$ & $\begin{array}{c}3 \sigma \text { Flux } \\
\text { Density }(\mu \mathrm{Jy})\end{array}$ & $\begin{array}{c}3 \sigma L_{\mathrm{R}} \\
\left(\mathrm{erg} \mathrm{s}^{-1}\right)\end{array}$ & $\begin{array}{c}\text { IMBH } \\
\text { Mass }\left(M_{\odot}\right)\end{array}$ & $\begin{array}{c}3 \sigma L_{\mathrm{X}} \\
\left(\mathrm{erg} \mathrm{s}^{-1}\right)\end{array}$ & $\begin{array}{l}\text { IMBH Mass } \\
\text { Fraction }\end{array}$ & $\begin{array}{c}\text { Core } \\
\text { Radius (") }\end{array}$ & $\begin{array}{l}\text { Brownian } \\
\text { Radius (") }\end{array}$ \\
\hline $\mathbf{M 3}^{\mathrm{a}}$ & 134211.38 & 282239.1 & 1.0 & 2 & 10.1 & 4 & $<5.8$ & $<3.5 \times 10^{27}$ & $<1460$ & $<7.8 \times 10^{30}$ & $<0.37$ & 22.2 & 0.28 \\
\hline M5 & 151833.21 & 020451.8 & 0.2 & 2 & 7.7 & 1 & $<4.2$ & $<1.5 \times 10^{27}$ & $<1060$ & $<3.0 \times 10^{30}$ & $<0.28$ & 26.4 & 0.38 \\
\hline M4 & 162335.03 & -263133.8 & 1.0 & 2 & 1.8 & 30 & $<5.3$ & $<1.0 \times 10^{26}$ & $<390$ & $<1.5 \times 10^{29}$ & $<0.40$ & 69.6 & 1.65 \\
\hline M107 & 163231.86 & -130313.6 & 0.1 & 1 & 6.1 & 4 & $<5.6$ & $<1.3 \times 10^{27}$ & $<990$ & $<2.4 \times 10^{30}$ & $<1.14$ & 33.6 & 0.50 \\
\hline M13 & 164141.21 & 362735.6 & 0.4 & 2 & 7.6 & 4 & $<4.9$ & $<1.7 \times 10^{27}$ & $<1110$ & $<3.4 \times 10^{30}$ & $<0.24$ & 37.2 & 0.52 \\
\hline M12 & 164714.18 & -015654.7 & 0.8 & 1 & 5.2 & 4 & $<4.3$ & $<7.0 \times 10^{26}$ & $<800$ & $<1.3 \times 10^{30}$ & $<0.92$ & 47.4 & 0.78 \\
\hline M10 & 16578.92 & -040558.0 & 1.0 & 13 & 4.4 & 6 & $<4.9$ & $<5.7 \times 10^{26}$ & $<740$ & $<1.0 \times 10^{30}$ & $<0.40$ & 46.2 & 0.80 \\
\hline M62 & 170112.98 & -300649.0 & 0.2 & 2 & 6.7 & 29 & $<6.6$ & $<1.8 \times 10^{27}$ & $<1130$ & $<3.6 \times 10^{30}$ & $<0.16$ & 13.2 & 0.18 \\
\hline M19 & 170237.80 & -261604.7 & 1.1 & 5 & 8.2 & 5 & $<4.8$ & $<1.9 \times 10^{27}$ & $<1170$ & $<4.0 \times 10^{30}$ & $<0.17$ & 25.8 & 0.35 \\
\hline N6304 & $1714 \quad 32.25$ & -292743.3 & 0.2 & 1 & 5.9 & 7 & $<5.8$ & $<1.2 \times 10^{27}$ & $<980$ & $<2.3 \times 10^{30}$ & $<0.35$ & 12.6 & 0.18 \\
\hline M92 & 171707.43 & 430809.26 & 0.1 & 2 & 8.9 & 1 & $<3.6$ & $<1.7 \times 10^{27}$ & $<1110$ & $<3.4 \times 10^{30}$ & $<0.41$ & 15.6 & 0.22 \\
\hline N6325 ${ }^{\mathrm{b}}$ & 171759.21 & -234557.6 & 2.0 & 6 & 6.5 & 8 & $<4.5$ & $<1.1 \times 10^{27}$ & $<960$ & $<2.2 \times 10^{30}$ & $<0.92$ & 1.8 & 0.03 \\
\hline M9 & 171911.78 & -183058.5 & 2.0 & 10 & 7.8 & 10 & $<3.8$ & $<1.4 \times 10^{27}$ & $<1030$ & $<2.7 \times 10^{30}$ & $<0.40$ & 27.0 & 0.40 \\
\hline Liller 1 & 173324.56 & $\begin{array}{lll}-33 & 23 & 22.4\end{array}$ & 0.3 & 3 & 8.1 & 12 & $<6.8$ & $<2.7 \times 10^{27}$ & $<1320$ & $<5.8 \times 10^{30}$ & $<0.20$ & 5.4 & 0.07 \\
\hline M14 & 173736.10 & -031445.3 & 0.5 & 6 & 9.3 & 6 & $<3.9$ & $<2.0 \times 10^{27}$ & $<1190$ & $<4.2 \times 10^{30}$ & $<0.15$ & 47.4 & 0.64 \\
\hline Terzan 5 & 174804.85 & -244644.6 & 1.0 & 11 & 5.9 & 19 & $<9.8$ & $<2.0 \times 10^{27}$ & $<1190$ & $<4.2 \times 10^{30}$ & $<0.21$ & 9.6 & 0.13 \\
\hline N6440 & 174852.70 & -20 2136.9 & 1.1 & 5 & 8.5 & 20 & $<6.5$ & $<2.8 \times 10^{27}$ & $<1340$ & $<6.1 \times 10^{30}$ & $<0.30$ & 8.4 & 0.11 \\
\hline $\mathrm{N} 5522^{\mathrm{b}}$ & $1803 \quad 34.89$ & $\begin{array}{lll}-30 & 02 & 03.2\end{array}$ & $\sim 2^{\mathrm{c}}$ & & 7.7 & 6 & $<9.5$ & $<3.4 \times 10^{27}$ & $<1430$ & $<7.4 \times 10^{30}$ & $<0.36$ & 3.0 & 0.04 \\
\hline N6539 & 180449.68 & -073509.1 & 0.32 & 6 & 7.8 & 20 & $<4.4$ & $<1.6 \times 10^{27}$ & $<1090$ & $<3.2 \times 10^{30}$ & $<1.22$ & 22.8 & 0.32 \\
\hline N6544 & 180720.12 & -245953.6 & 0.95 & 8 & 3.0 & 20 & $<5.3$ & $<2.9 \times 10^{26}$ & $<570$ & $<4.7 \times 10^{29}$ & $<0.90$ & 3.0 & 0.06 \\
\hline M28 & 182432.73 & -245213.0 & 0.7 & 2 & 5.5 & 20 & $<5.1$ & $<9.2 \times 10^{26}$ & $<890$ & $<1.8 \times 10^{30}$ & $<0.24$ & 14.4 & 0.23 \\
\hline M22 & 183623.94 & -235417.1 & 0.8 & 1 & 3.1 & 28 & $<5.0$ & $<2.9 \times 10^{26}$ & $<570$ & $<4.7 \times 10^{29}$ & $<0.14$ & 79.8 & 1.56 \\
\hline N6712 & 185304.30 & -084222.0 & 0.5 & 9 & 8.0 & 9 & $<4.8$ & $<1.8 \times 10^{27}$ & $<1150$ & $<3.8 \times 10^{30}$ & $<0.90$ & 45.6 & 0.63 \\
\hline M54 & 185503.33 & -302847.5 & 0.1 & 1 & 23.9 & 15 & $<7.1$ & $<2.4 \times 10^{28}$ & $<2990$ & $<6.1 \times 10^{31}$ & $<0.21$ & 5.4 & 0.04 \\
\hline N6760 & 191112.01 & 010149.7 & 0.5 & 6 & 7.4 & 20 & $<4.5$ & $<1.5 \times 10^{27}$ & $<1060$ & $<3.0 \times 10^{30}$ & $<0.42$ & 20.4 & 0.29 \\
\hline M55 & 193959.71 & -305753.1 & 0.8 & 1 & 5.7 & 4 & $<5.1$ & $<1.0 \times 10^{27}$ & $<910$ & $<1.9 \times 10^{30}$ & $<0.48$ & 108.0 & 1.67 \\
\hline $\mathrm{M} 15^{\mathrm{b}}$ & 212958.33 & 121001.2 & 0.2 & 1 & 10.3 & 1 & $<6.3$ & $<4.0 \times 10^{27}$ & $<1530$ & $<9.0 \times 10^{30}$ & $<0.34$ & 8.4 & 0.10 \\
\hline M2 & 213326.96 & -004922.9 & 1.0 & 12 & 11.5 & 6 & $<3.9$ & $<3.1 \times 10^{27}$ & $<1390$ & $<6.8 \times 10^{30}$ & $<0.24$ & 19.2 & 0.24 \\
\hline $\mathbf{M 3 0}^{\mathrm{b}}$ & 214022.12 & -231047.5 & 0.1 & 1 & 8.6 & 4 & $<3.9$ & $<1.7 \times 10^{27}$ & $<1120$ & $<3.5 \times 10^{30}$ & $<0.84$ & 3.6 & 0.05 \\
\hline
\end{tabular}

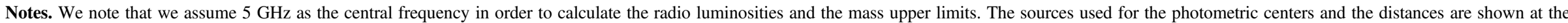
bottom of the table. The core radius of Liller 1 is adopted from Saracino et al. (2015), while we use Harris (2010) for the rest of the clusters.

${ }^{\mathrm{a}} \mathrm{GCs}$ used for the stacking analysis are indicated in boldface.

${ }^{\mathrm{b}}$ Core collapsed GCs as adapted by Trager et al. (1995) catalog.

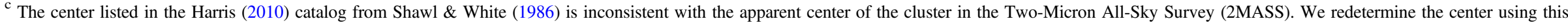
2MASS image, and this value is the one listed in Table 1. The uncertainty in the value is not well determined, but we estimate $\sim 2^{\prime \prime}$.

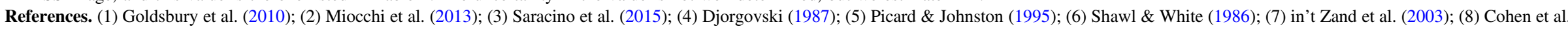

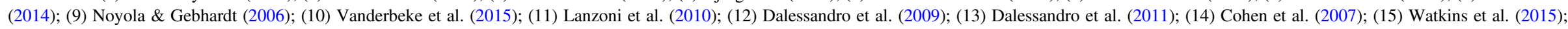

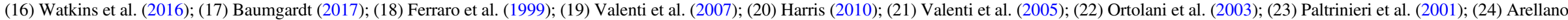
Ferro et al. (2013); (25) Ortolani et al. (2007); (26) Saracino et al. (2015); (27) Ortolani et al. (1999); (28) Kunder et al. (2013); (29) Contreras et al. (2010); (30) Kaluzny et al. (2013). 


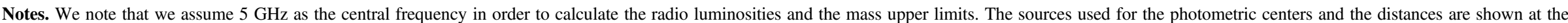
bottom of the table. The core radii are adopted from Harris (2010).

${ }^{\mathrm{a}} \mathrm{GCs}$ used for the stacking analysis are indicated in boldface.

b Core collapsed GCs as adapted by Trager et al. (1995) catalog.

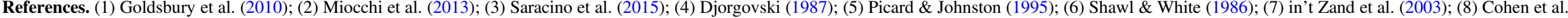

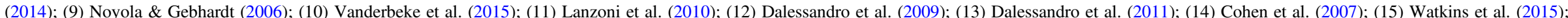

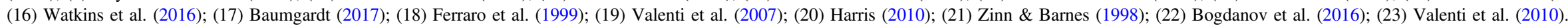




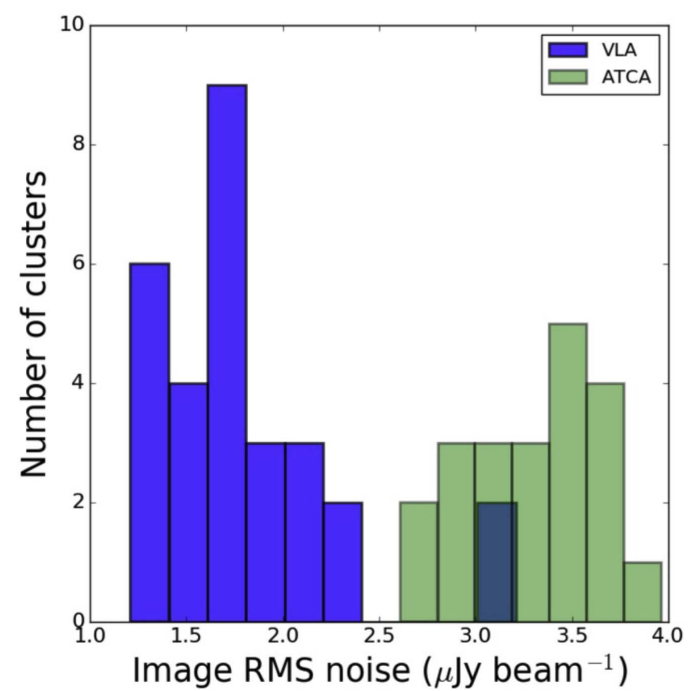

Figure 1. Distribution of the observed rms noise in our frequency-averaged images for 50 Galactic GCs. VLA data are blue and ATCA data are green.

VLA, the two frequency bands were imaged separately and with a Briggs robust value of 1 . The median synthesized beam in the $5.5 \mathrm{GHz}$ images (and in the frequency-averaged images) is 3 !" $2 \times 1$ !" 6 . The median on-source integration time was $17.0 \mathrm{hr}$, yielding median rms noise levels of 4.1 and $4.6 \mu \mathrm{Jy} \mathrm{bm}^{-1}$ in the 5.5 and $9.0 \mathrm{GHz}$ bands, respectively. As with the VLA images, we averaged images in the two frequency bands together, and the median rms was $3.3 \mu \mathrm{Jy} \mathrm{bm}^{-1}$ in the resulting $7.25 \mathrm{GHz}$ images. This is about twice the median rms of the VLA images (Figure 2) but still very sensitive, allowing tight constraints on the presence of central radio sources. The distribution of the observed rms of the ATCA images (Figure 3) is shown in Figure 1.

Five GCs in the sample were observed by both VLA and ATCA; for all but NGC 6522 (which had only $2.5 \mathrm{hr}$ on-source with the VLA) the beam size is smaller and the rms is substantially lower in the VLA images, so we use the VLA data for subsequent analysis.

\subsection{Stacking Analysis}

We also stacked all the clusters to give the highest sensitivity to low-level emission under the assumption that IMBHs exist in the centers of GCs. For the stack, we only used clusters that do not have any central radio emission (from unresolved pulsars or bright $\mathrm{X}$-ray binaries), in order to make a weighted-mean image of these fields. In total, we used 24 clusters from VLA and 14 from ATCA observations, which are noted in boldface in Tables 1 and 2 . Twelve clusters that were excluded from the deep stack are discussed extensively in Sections 3.3 and 4.2.

To do this, the frequency-averaged images were convolved to a common resolution (2". 2 for VLA data and 6". 0 for ATCA data) using the AIPS tasks CONVL. Images were aligned so that the cluster centers aligned at the image center (see Tables 1 and 2 for GC centers). We then used the AIPS task STACK to produce the weighted mean image (see Lindroos et al. 2015 for details of the stacking technique). Separate stacks were made for VLA (Figure 4) and VLA (Figure 5) clusters (see Section 4.2).

\section{Data Analysis}

\subsection{Linking IMBH Mass and Radio Luminosity}

The "fundamental plane" of BHs describes the relation between X-ray luminosity $\left(L_{\mathrm{X}}\right)$, radio luminosity $\left(L_{\mathrm{R}}=4 \pi d^{2} \nu S_{\nu}\right)$, and the mass of the $\mathrm{BH}\left(M_{\mathrm{BH}}\right)$ for objects ranging from stellar-mass BHs to supermassive BHs (Merloni et al. 2003; Falcke et al. 2004; Plotkin et al. 2012). It implies that accreting BHs of all masses show a correlation between X-ray and radio luminosity but that more massive BHs have a higher ratio of $L_{\mathrm{R}} / L_{\mathrm{X}}$ at a fixed $L_{\mathrm{X}}$.

Since no IMBHs have been dynamically confirmed, their consistency with the fundamental plane has not been tested: their behavior is an interpolation between the extreme low and high $\mathrm{BH}$ masses. For this paper, we assume that accreting IMBHs would behave in a manner consistent with the fundamental plane, and we use our radio observations to constrain the masses of IMBHs at the centers of Galactic GCs.

We adopt the form of the fundamental plane with $\mathrm{BH}$ mass as the dependent variable (Miller-Jones et al. 2012; Plotkin et al. 2012):

$$
\begin{aligned}
\log M_{\mathrm{BH}}= & (1.638 \pm 0.070) \log L_{\mathrm{R}} \\
& -(1.136 \pm 0.077) \log L_{\mathrm{X}} \\
& -(6.863 \pm 0.790)
\end{aligned}
$$

where the black hole mass $M_{\mathrm{BH}}$ is given in units of $M_{\odot}$, and $L_{\mathrm{X}}$ and $L_{\mathrm{R}}$ are given in erg s${ }^{-1}$. This form of the fundamental plane is derived using BHs in the "low/hard" accretion state $(\lesssim$ a few percent of the Eddington rate), in which the X-ray emission is thought to originate in a corona or possibly near the base of the jet while the radio emission is partially self-absorbed synchrotron radiation from the jet. If IMBHs are present in GCs and accreting, then they must be in the low state or else they would be easily observed as bright X-ray sources. The respective luminosities in this plane are formally defined between $0.5-10 \mathrm{keV}\left(L_{\mathrm{X}}\right)$ and at $5 \mathrm{GHz}\left(L_{\mathrm{R}}\right)$. In all cases we assume flat radio spectra consistent with that observed for lowluminosity active galactic nuclei (AGNs), e.g: Nagar et al. (2000) (see also Blandford \& Königl 1979; Hjellming \& Johnston 1988; Gallo et al. 2005) to translate the observed flux density $\left(L_{\mathrm{R}}\right)$ limits into $5 \mathrm{GHz}$ luminosities.

Consistent with previous work, we assume that the putative IMBH accretes from ambient intracluster gas in a manner similar to low-luminosity central supermassive BHs in galaxies (Maccarone 2003; Pellegrini 2005; Strader et al. 2012; Haggard et al. 2013). In GCs, gas should be present due to the high density of red giants and their associated winds. Direct evidence of this gas has been seen in 47 Tuc and M15, revealed by the radial distribution of the dispersion measure of pulsars, which traces the free electron density inside the GC (Freire et al. 2001). These observations and the lack of $\mathrm{HI}$ in GCs implies that the gas must be mostly ionized (van Loon et al. 2006); here we assume that intracluster gas is evenly distributed and is fully ionized and isothermal at a temperature of $T=10^{4} \mathrm{~K}$. We assume a gas number density of $n=0.2$ $\mathrm{cm}^{-3}$, corresponding to an electron density of $n_{e}=0.1 \mathrm{~cm}^{-3}$, consistent with the aforementioned pulsar data and expectations for freely expanding red giant winds (Pfahl \& Rappaport 2001; Pooley \& Rappaport 2006).

The Bondi accretion rate for an isothermal gas onto a $\mathrm{BH}$ is given by $\dot{M}_{\mathrm{Bondi}}=e^{3 / 2} \pi G^{2} M^{2} \rho c_{s}^{-3}$, where $e^{3 / 2}=$ $2(5-3 \gamma)^{(5-3 \gamma) / 2(\gamma-1)}$, from $\gamma=1$ for an isothermal gas; 
$G$ is the gravitational constant; $M$ is the BH mass; and $\rho=n \mu$ $m_{H}$ is the gas density (Bondi 1952). The sound speed $c_{s}$ in an isothermal gas is given by $c_{s}=\left(k_{B} T / \mu m_{H}\right)^{1 / 2}$ for the mean molecular mass $\mu$. Here we take $\mu=0.59$, appropriate for ionized gas with the usual composition of a GC (Fall \& Rees 1985). Substituting in typical values, the Bondi rate in units of $\mathrm{g} \mathrm{s}^{-1}$ is

$$
\dot{M}_{\text {Bondi }}=1.18 \times 10^{17}\left(\frac{M}{2000 M_{\odot}}\right)^{2}\left(\frac{n}{0.2 \mathrm{~cm}^{-3}}\right)\left(\frac{T}{10^{4} \mathrm{~K}}\right)^{-3 / 2} .
$$

To yield the Bondi rate in units of $M_{\odot} \mathrm{yr}^{-1}$, the pre-factor on the above equation is instead $1.88 \times 10^{-9}$.

The results of Pellegrini (2005) show that low-luminosity AGNs are not as luminous as would be expected if they were accreting at the Bondi rate in a radiatively efficient manner (see also recent theoretical work on sub-Bondi accretion by Inayoshi et al. 2018). The quantity directly constrained by X-ray measurements is not the accretion rate itself but a combination of the radiative efficiency and the accretion rate. The X-ray luminosity is given by the standard equation $L_{\mathrm{X}}=\epsilon \dot{M} c^{2}$, for the radiative efficiency $\epsilon$ and the speed of light $c$. As in our previous work (Maccarone \& Servillat 2008; Strader et al. 2012), we assume that the accretion flow is radiatively inefficient, as for example in an advection-dominated accretion flow (Narayan \& Yi 1995), such that the radiative efficiency $\epsilon=\eta\left(\dot{M} / \dot{M}_{\text {edd }}\right)$ for some normalization $\eta$ and $\dot{M}_{\text {edd }}$ is the Eddington accretion rate.

If we parameterize the accretion rate $\dot{M}$ as a fraction $f_{b}$ of the Bondi rate (so that $\dot{M}=f_{b} \dot{M}_{\text {Bondi }}$ ), then the observationally determined quantity is $\eta f_{b}^{2}$. Using the $\dot{M}_{\text {Bondi }}$ and $L_{\mathrm{X}}$ measurements of Pellegrini (2005) for low-luminosity AGNs, and adjusting for their use of $\gamma=4 / 3$ rather than our isothermal $\gamma=1$, we find that the quantity $\eta f_{b}^{2}$ has a median of about 0.005 but an extremely large spread of about $1.5 \mathrm{dex}$ in the log. Considering its large uncertainty, this value is equivalent to the $\eta f_{b}^{2}=0.0045$ implicitly used in Strader et al. (2012), and for consistency we use this latter value. We discuss the uncertainty in this assumption and its implications for the mass limits below (Section 3.1.1).

Measuring the upper limits on the radio luminosity, $L_{\mathrm{R}}$, for a GC IMBH, we use the fundamental plane assuming that the $\mathrm{BH}$ follows the $L_{\mathrm{R}}-L_{\mathrm{X}}$ relationship and the accretion efficiency in order to determine the predicted $L_{\mathrm{X}}$ and subsequently to constrain the $M_{\mathrm{BH}}$. We emphasize that our methodology uses predicted $L_{\mathrm{X}}$ rather than observed $L_{\mathrm{X}}$ in the context of the fundamental plane to obtain IMBH mass constraints. This is because most GCs in our sample do not have published X-ray limits on a central IMBH. For one cluster ( $\omega$ Cen) the published $\mathrm{X}$-ray limit is more stringent than the limit inferred from our formalism (see Section 5.2.1). Given the scatter in the fundamental plane, additional observed X-ray limits would also provide valuable constraints on the presence of IMBHs in our GC sample, and our team is currently in the process of conducting a uniform X-ray analysis of the sample.

For the rest of this paper, we use $3 \sigma$ upper limits as a constraint on the radio luminosity to constrain the IMBH mass. The plane between $M_{\mathrm{BH}}$ (in $M_{\odot}$ ), the distance $d$ (in kpc), and the observed $5 \mathrm{GHz}$ flux density or upper limit $S$ (in $\mu \mathrm{Jy}$ ) implied by the above formalism is $\log M_{\mathrm{BH}}=0.743 \log d+$ $0.372 \log S+2.134$.

\subsubsection{Uncertainties in This Formalism}

As discussed in Strader et al. (2012), the main uncertainty in the mass limits predicted by this formalism is the combination of the radiative efficiency and the actual accretion rate, here parameterized by $\eta f_{b}^{2}$. The scatter in the fundamental plane itself is nearly negligible. The observational scatter of about 1.5 dex in $\eta f_{b}^{2}$ translates into a 0.39 dex scatter in $\log M_{\mathrm{BH}}$. Another source of uncertainty is the gas density $\rho$, but this is much harder to quantify given the scarcity of observational data. An uncertainty of 0.3 dex in $\log \rho$, suggested by the few Galactic GC data points as well as the theoretical considerations discussed above, barely increases the overall uncertainty in $\log M_{\mathrm{BH}}$ (to $0.43 \mathrm{dex}$ ). But we emphasize that the distribution of $\rho$ is not well constrained.

A systematic uncertainty is our assumption of an isothermal gas rather than an adiabatic gas or one with an intermediate index. The isothermal assumption is conservative: it produces higher limits on $M_{\mathrm{BH}}$ than other choices. If we instead used the opposite extreme, then an adiabatic gas with $\gamma=5 / 3$, $\log M_{\mathrm{BH}}$ would be lower by $0.52 \mathrm{dex}$. This is of the same order as the variation due to $\eta f_{b}^{2}$, though the quantities are not necessarily independent.

The above discussion illustrates that the exact predictions depend on the assumptions made and that these limits should not be compared to other radiative limits on X-ray or radio emission without an appropriate consideration of the assumptions.

\subsection{IMBH Location}

We adopt the photometric center of each GC as the best current estimate of its center of mass. These are listed in Tables 1 and 2. The photometric centers come primarily, though not exclusively, from fitting models to Hubble Space Telescope (HST) star count observations. For example, Goldsbury et al. (2010) determined the photometric centers of 65 Milky Way GCs by performing an analysis of star counts in HST/Advanced Camera for Survey images, while Miocchi et al. (2013) supplemented HST observations with groundbased data.

Dynamical friction leads an IMBH to spiral to the center of mass of its host cluster on a short timescale (Matsubayashi et al. 2007). From this location, encounters with stars or stellar remnants can perturb the $\mathrm{IMBH}$, especially if the $\mathrm{BH}$ mass is relatively low. Using the principle of Brownian motion, Chatterjee et al. (2002) analyzed the dynamics in the context of a $\mathrm{BH}$ at the center of a dense stellar cluster. Assuming a Plummer potential for the stellar system surrounding the central $\mathrm{BH}$, the predicted variance of mean-squared one-dimensional deviations will be $\left\langle x^{2}\right\rangle=(2 / 9)\left(M_{*} / M_{\mathrm{BH}} r_{c}^{2}\right)$, where $M_{*}$ is the average mass of a star in the cluster core $\left(\sim 1 M_{\odot}\right)$ and $r_{c}$ is the cluster core radius (see also Strader et al. 2012). The core of a GC can be depleted of lower-mass stars due to mass segregation; therefore, we adopt a conservative value for the mass of the observable stars in the core of GCs (Fregeau et al. 2002). The actual motion of an IMBH would depend of course on the detailed mass profile of the inner regions, but this basic estimate gives a guide to how much the IMBH might wander as a function of cluster parameters. To calculate the Brownian radii for our GCs, we use the $3 \sigma$ mass limits as given in Tables 1 and 2. For GCs with a cluster centroid uncertainty that 
is larger than the Brownian radii, we use the former for our analysis.

We note that when the current paper was close to submission, de Vita et al. (2018) published a more sophisticated model, based on $N$-body simulations, for predicting the random motion of IMBHs in GC centers. Nonetheless, their results are generally consistent with the simple Brownian motion model we use.

\subsection{Notes on Clusters with Radio Sources near Their Centers}

Our basic result is that we find no compelling evidence for accreting IMBHs in any of the $50 \mathrm{GCs}$ in our sample. However, some clusters do have radio sources near their photometric centers, which are associated with X-ray binaries or pulsars. Here we discuss these sources and why we favor non-IMBH explanations for the emission in all cases. Furthermore, previous studies regarding the IMBH existence in individual clusters are discussed extensively in Section 5.2.

\subsubsection{Liller 1}

This massive dense cluster shows several steep spectrum sources near the center (but outside the Brownian radius of a putative IMBH). Given the high interaction rate inferred for Liller 1 (Saracino et al. 2015) and the strong steep-spectrum emission as identified by Fruchter \& Goss (2000), these sources are likely to be pulsars. Their presence does not affect the IMBH limits for the cluster, but we exclude it from the VLA stack.

\subsection{2. $M 15$}

Our nondetection of an IMBH in M15 has previously been published in Strader et al. (2012). As discussed in that work, there are two previously known radio bright X-ray binaries and a pulsar near the cluster center (within 4"; Wolszczan et al. 1989; Johnston et al. 1991; Miller-Jones et al. 2011). However, there is no radio emission at the center of M15 within the Brownian radius expected for an IMBH. See Strader et al. (2012) for more discussion.

\subsection{3. $M 62$}

M62 has six pulsars known in its core (D'Amico et al. 2001; Possenti et al. 2003; Lynch et al. 2012), two within 2". 5 of the cluster center. One of these, $\mathrm{J} 1701-3006 \mathrm{~F}$, is a $4.5 \sigma$ detection in our $4.7 \mathrm{GHz}$ image but is not detected at $7.4 \mathrm{GHz}$. There is no significant detection at either frequency within the Brownian radius of an IMBH.

\subsubsection{NGC 6388}

NGC 6388 was previously observed with ATCA to search for an IMBH, and no central radio emission was detected to a level $<42 \mu \mathrm{Jy}$ at $5.5 \mathrm{GHz}$ (Bozzo et al. 2011).

In our ATCA $5.5 \mathrm{GHz}$ image, there is a source located near the cluster center with the flux density $20.2 \pm 3.6 \mu \mathrm{Jy}$ and a J2000 location of (R.A., decl.) $=(17: 36: 17.276,-44: 44: 09.03)$, just 1 ." 1 from the cluster photometric center (Figure 3 ). The source is not significantly detected in the $9.0 \mathrm{GHz}$ image, with a flux density at its location of $4.6 \pm 3.6 \mu \mathrm{Jy}$. If we conservatively assume a uniform prior on $\alpha$ between -3 and 3 and use the $3 \sigma$ upper limit of the $9.0 \mathrm{GHz}$ flux density $(<10.8 \mu \mathrm{Jy})$, then the most likely value of $\alpha$ is -2.1 , with a $3 \sigma$ upper limit to the spectral index of -0.2 . Therefore the most probable interpretation is that this central source is a pulsar in the cluster, but a flat-spectrum source cannot be definitively ruled out.

The presence of a pulsar near the cluster center would be far from surprising, as a large pulsar population is expected in NGC 6388 based on the Fermi/LAT detection of GeV $\gamma$-rays (Abdo et al. 2010), its high stellar encounter rate (Bahramian et al. 2013), and its large X-ray source population (Bozzo et al. 2011; Maxwell et al. 2012). In addition, the centroid uncertainty for an IMBH of mass $>1000 M_{\odot}$ in NGC 6388 is $\lesssim 0$ ". 3 . We therefore conclude that the central point source is not an IMBH but could be a pulsar. We proceed by using the 9.0 $\mathrm{GHz}$ image to constrain the mass of an IMBH; the limit listed in Table 2 corresponds to the sensitivity of the $9.0 \mathrm{GHz}$ image alone.

\subsubsection{NGC 6624}

The center of the cluster NGC 6624 has two neutron star sources that emit in the radio: the bright pulsar PSR 1820-30A and the ultracompact low-mass X-ray binary 4U 1820-30 (Figure 6). Radio timing observations of the pulsar and X-ray timing of the X-ray binary have been used in a number of recent papers to argue for the presence of an IMBH (Peuten et al. 2014; Perera et al. 2017), though Gieles et al. (2018) argue that standard dynamical models of this GC can explain these observations without a massive $\mathrm{BH}$.

Consistent with previous work, we observe a bright radio source near the center of NGC 6624 (Figure 6). Using these ATCA data and new HST observations, we find that all of the radio emission observed is consistent with being from the X-ray binary $4 \mathrm{U}$ 1820-30. We also note that this is in agreement with the Migliari et al. (2004) finding that 4U 182030 dominates above $2 \mathrm{GHz}$, and the pulsar below that. As the level of detail necessary to reach this conclusion is out of the main line of our paper, we place most of it in the Appendix and here focus only on the radio emission from an IMBH.

To search for residual emission from a possible IMBH, we subtracted the X-ray binary 4U 1820-30 from each of the 5.5 and $9.0 \mathrm{GHz}$ images, assuming it was a point source. We fit a Gaussian component in the image-plane using the AIPS task JMFIT and then subtracted it, without any assumption on the spectral index value. No residuals are apparent in either image after the subtraction, suggesting that this assumption is reasonable. We remeasured the rms noise in a 24 " box around the cluster center after the subtraction, using this value for the IMBH limits. We find an rms noise of $4.2 \mu \mathrm{Jy} \mathrm{bm}^{-1}$ at $5.5 \mathrm{GHz}, 3.9 \mu \mathrm{Jy} \mathrm{bm}^{-1}$ at $9.0 \mathrm{GHz}$, and $3.3 \mu \mathrm{Jy} \mathrm{bm}^{-1}$ in the averaged image at $7.25 \mathrm{GHz}$. We use this latter limit to determine the IMBH mass limit using our standard formalism.

\subsection{6. $N G C 6652$}

NGC 6652 has a high central density, suggesting the efficient production of dynamically formed compact object binaries (Stacey et al. 2012), and several relatively bright X-ray sources have been detected by Chandra in this cluster. Only one radio pulsar has yet been detected in this cluster (DeCesar et al. 2015), but NGC 6652 is detected with Fermi/LAT at $\mathrm{GeV}$ energies, suggesting a substantial total population of millisecond pulsars (Abdo et al. 2010).

We detect a steep spectrum source in the ATCA image (present at $5.5 \mathrm{GHz}$ and absent at $9.0 \mathrm{GHz}$ ) that is offset by 

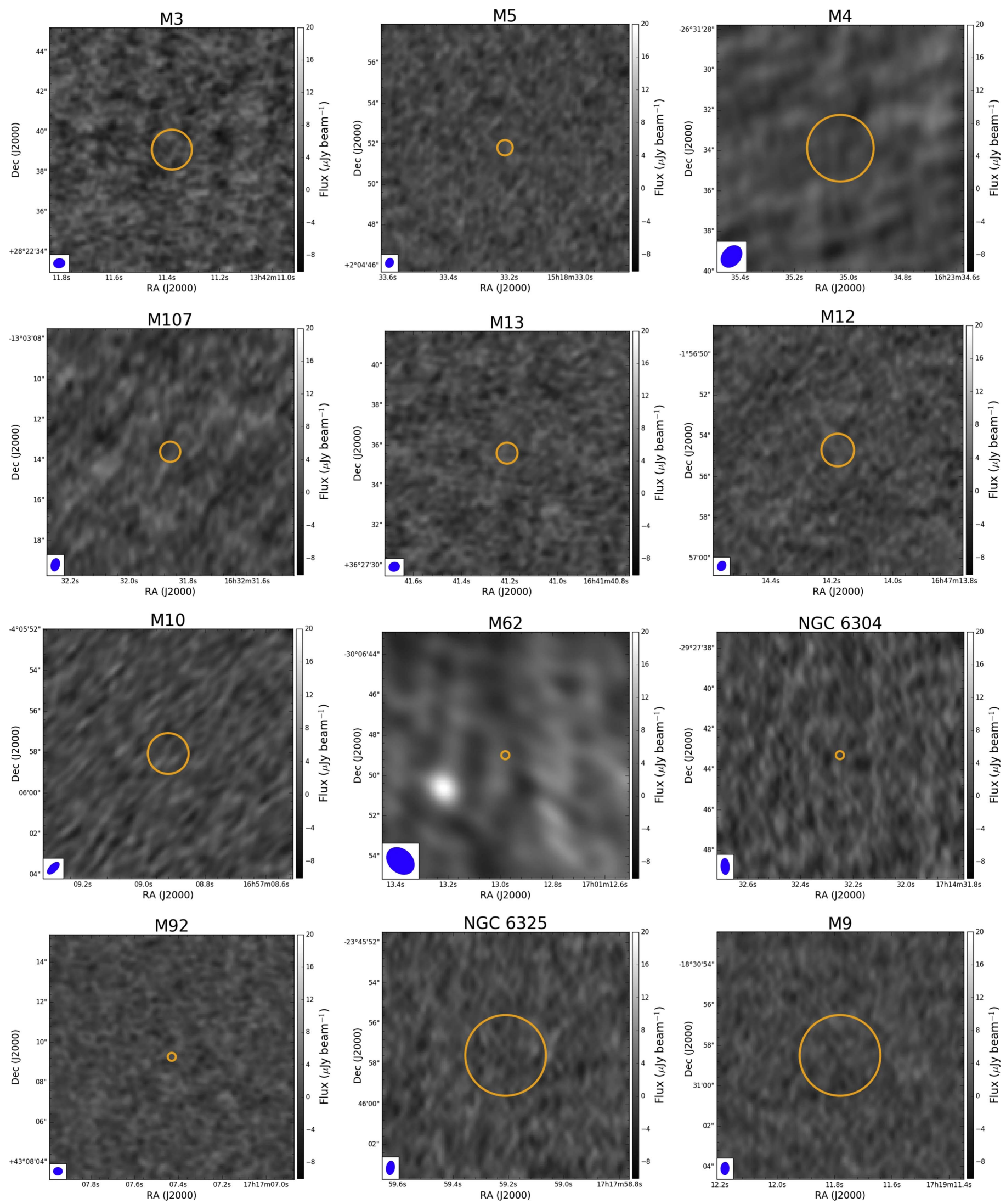

Figure 2. VLA frequency-averaged images of the GCs listed in Table 1, showing the central 12 !! $2 \times 12$ !' 2 for each cluster. The position of the cluster center is marked as an orange circle, and its size is matched to the wander radius of a putative IMBH. Synthesized beams are shown in blue in the bottom-left corner of all images. 

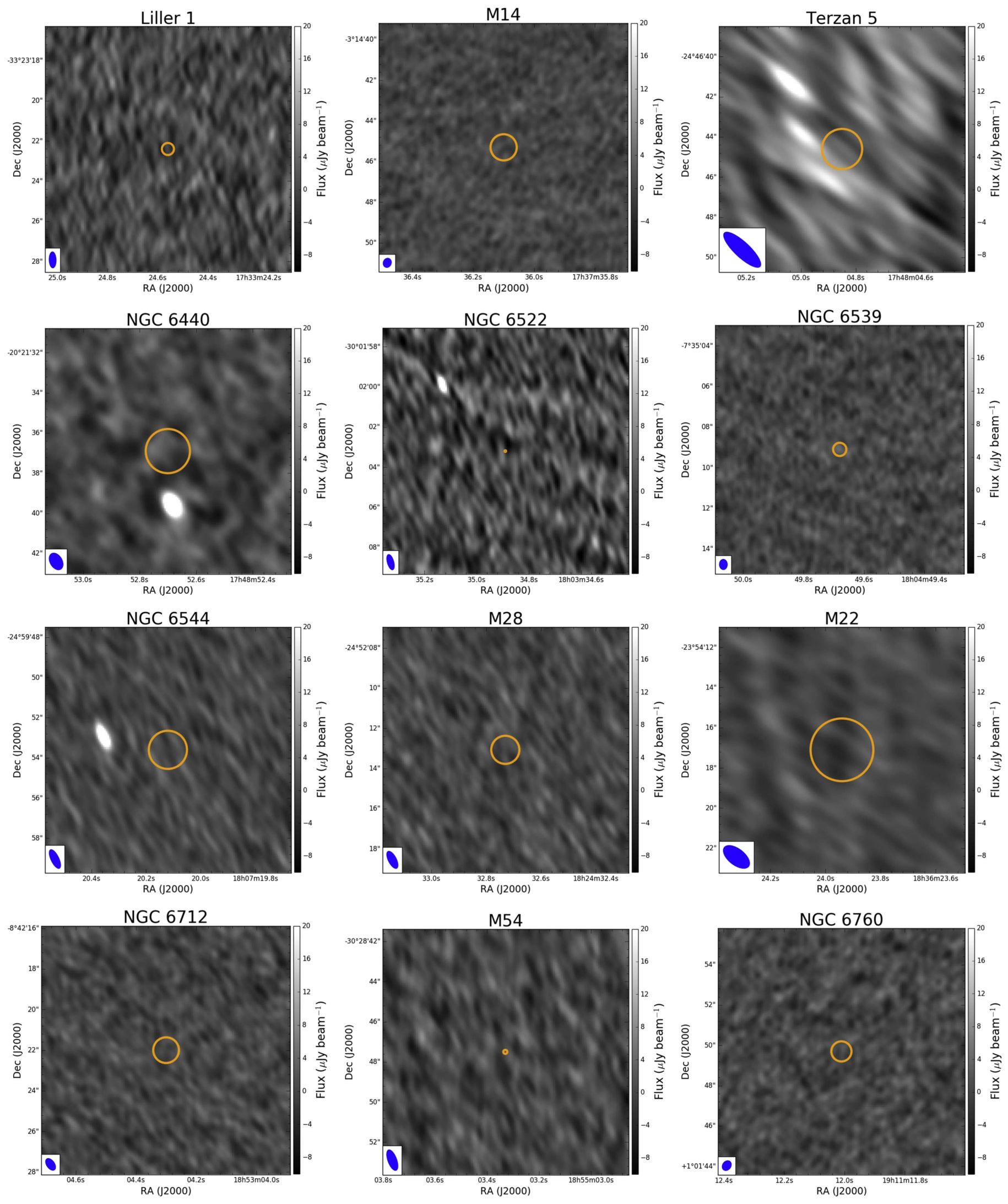

Figure 2. (Continued.) 

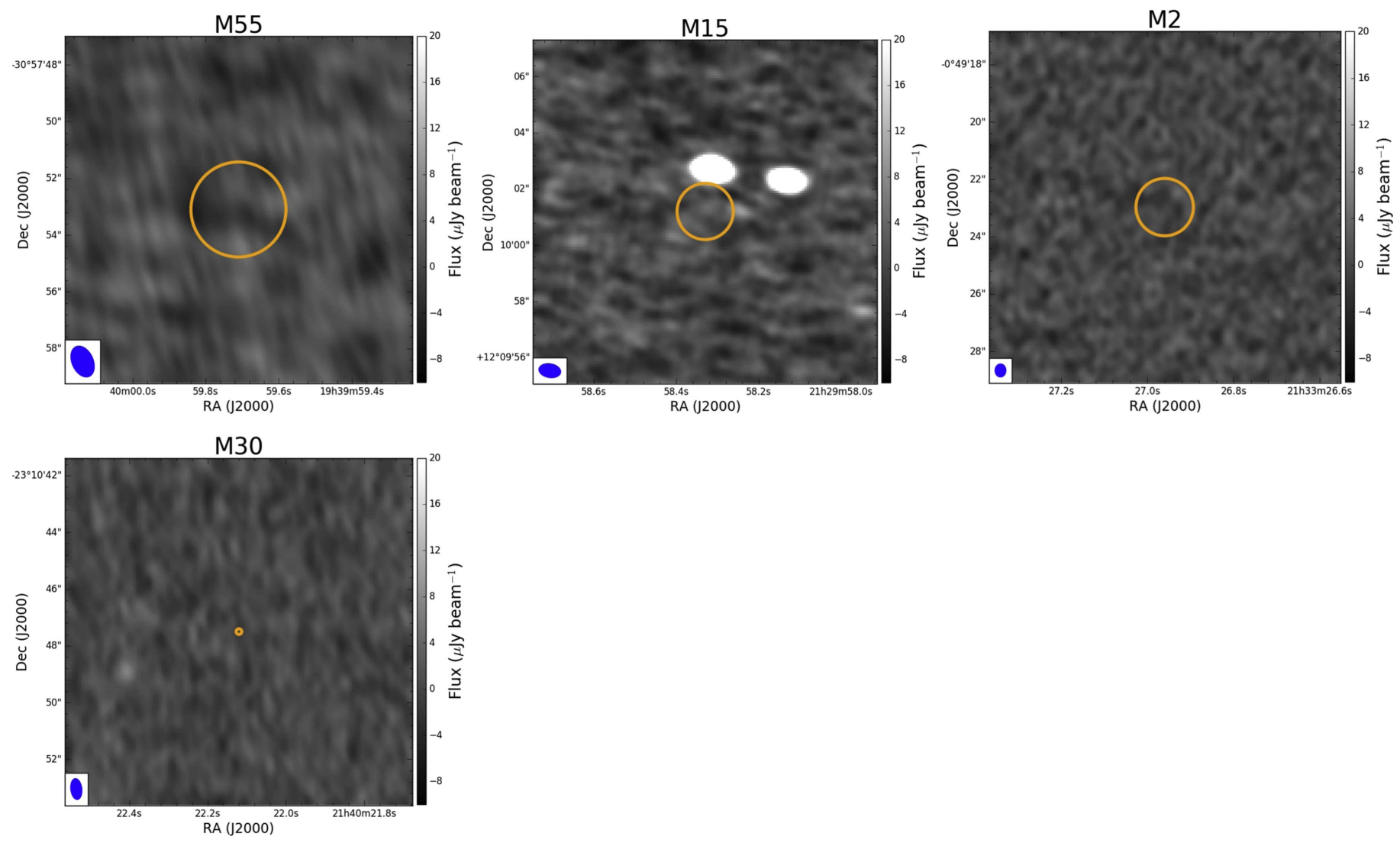

Figure 2. (Continued.)

about 1". 5 from the cluster center, so we can conclude that it is not associated with an IMBH. Just as for the central source in NGC 6388, the inferred steep spectrum implies that this source is likely a pulsar. This radio source is also 1 ." 5 from the nearest X-ray source in the cluster (source D of Stacey et al. 2012), ruling out an association. To place limits on the presence of an IMBH in NGC 6652, we only use the $9.0 \mathrm{GHz}$ image, which does not show emission from this source.

\subsubsection{Terzan 1}

There is a radio source near the center of Terzan 1 with position J2000 (R.A., decl.) $=(17: 35: 47.204,-30: 28: 54.89)$. This source may be associated with the quiescent X-ray binary CX2 (Cackett et al. 2006). However, we measure flux densities of $90.5 \pm 4.1 \mu \mathrm{Jy}$ at $5.5 \mathrm{GHz}$ and $34.5 \pm 4.1 \mu \mathrm{Jy}$ at $9.0 \mathrm{GHz}$, implying a steep spectral index of $\alpha=-2.0 \pm 0.3$, more consistent with a pulsar than an X-ray binary. We will revisit this source in future work; in any case, the steep spectrum is inconsistent with the expectations for low-level accretion onto an IMBH.

To search for any residual emission that may be present from an IMBH, we subtracted this source from the 5.5 and $9.0 \mathrm{GHz}$ images under the assumption that it is a point source, applying the same technique as in NGC 6624. No residuals are apparent in the $5.5 \mathrm{GHz}$ image. In the $9.0 \mathrm{GHz}$ image, a second source is visible at the $\sim 3 \sigma$ level (in fact, this source is approximately one beam away from the brighter source in the original 9.0 GHz image and is clearly detectable there as well). This source is $3^{\prime \prime}$ from the cluster center, far outside the Brownian radius of a $>1000 M_{\odot} \mathrm{IMBH}$, and hence cannot be attributed to an IMBH.

We re-estimate the rms noise from these residual images in a region $24^{\prime \prime}$ wide centered on the cluster center. We find rms sensitivities of $4.8 \mu \mathrm{Jy} \mathrm{beam}^{-1}$ at $5.5 \mathrm{GHz}, 4.1 \mu \mathrm{Jy}$ beam $^{-1}$ at $9.0 \mathrm{GHz}$, and $3.9 \mu \mathrm{Jy}$ beam $^{-1}$ in the combined frequency image and use these revised values for our analysis.

\subsubsection{Terzan 5}

Terzan 5 hosts a large population of pulsars, with 38 known in the cluster core (Ransom et al. 2005; Prager et al. 2017; Cadelano et al. 2018, P. Freire's "Pulsars in Globular Clusters" page 7 , and our images show many point sources. However, assuming a 1 " uncertainty on the position of the cluster center and an IMBH Brownian radius of 0 ". 12 , we find no VLA radio sources located right at the cluster center. ATCA images are also consistent with no flux at the cluster center but are less constraining because of the lower image resolution and poorer sensitivity.

\subsubsection{Terzan 6}

Terzan 6 hosts the known transient neutron star X-ray binary GRS 1747-312 near its center (at a projected distance of 0!" 8; Predehl et al. 1991; Pavlinsky et al. 1994; in't Zand et al. 2003). This source was in outburst and radio bright during our observation (its X-ray/radio correlation will be reported elsewhere), measured at $21.4 \pm 5.1 \mu \mathrm{Jy}$ in our ATCA $5.5 \mathrm{GHz}$ image and $17.3 \pm 5.3 \mu \mathrm{Jy}$ in the $9.0 \mathrm{GHz}$ image. We

7 http://www.naic.edu/ pfreire/GCpsr.html 

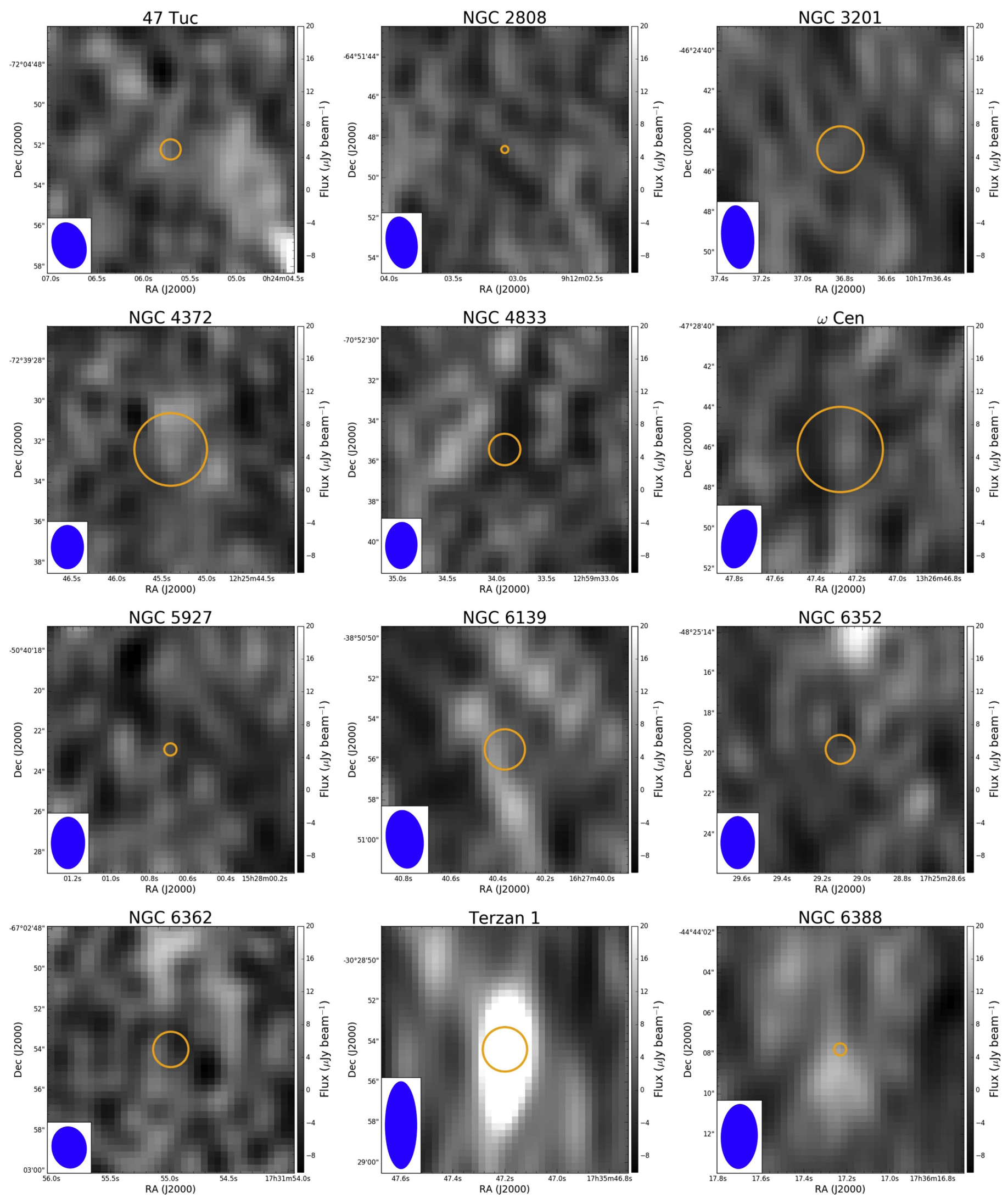

Figure 3. ATCA frequency-averaged images of the GCs listed in Table 2. See Figure 2 for more details

subtracted this point source, and there is no residual emission near the cluster center. We remeasured the rms sensitivities of these residual images in a $24^{\prime \prime}$ region and found
$5.3 \mu \mathrm{Jy}$ beam $^{-1}$ at $5.5 \mathrm{GHz}, 5.3 \mu \mathrm{Jy}$ beam $^{-1}$ at $9.0 \mathrm{GHz}$, and $4.2 \mu \mathrm{Jy}$ beam $^{-1}$ in the frequency-averaged image, which we use for our IMBH limits. 

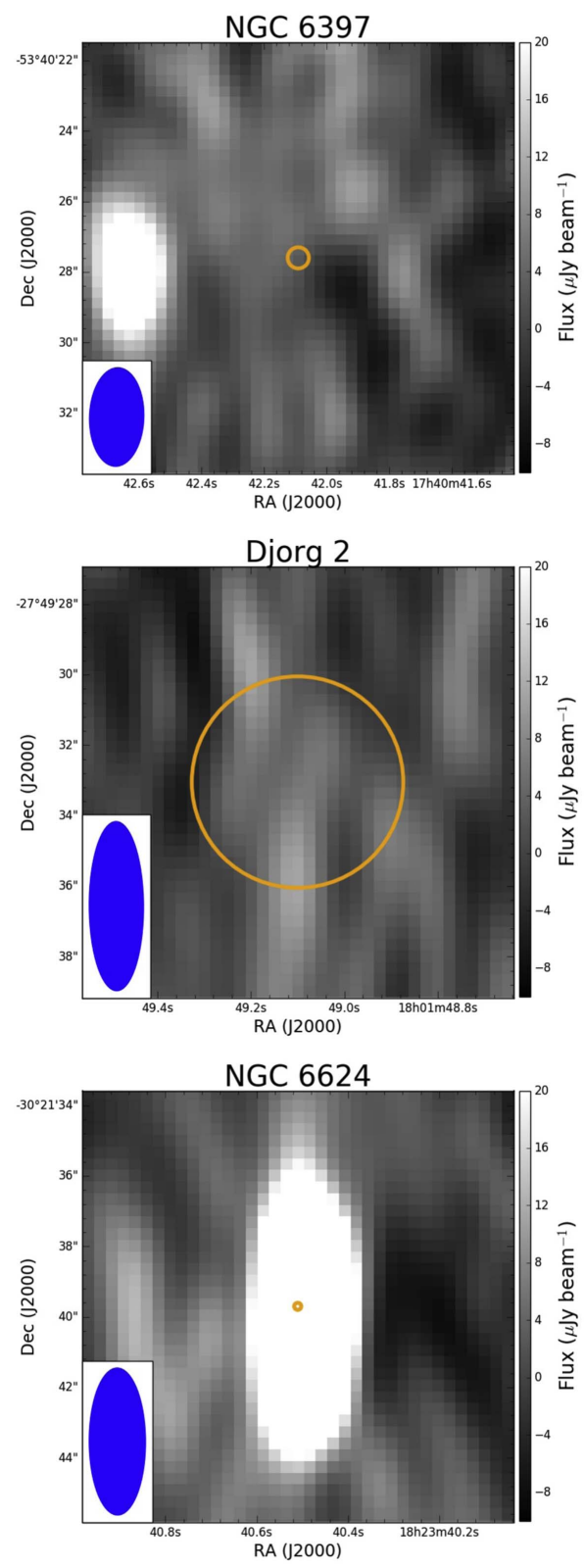

\section{Results}

\subsection{IMBH Mass Limits per Cluster}

We followed the method described in Section 3.2 to search for IMBHs in cluster centers. The clusters discussed in Section 3.3 were considered individually, as detailed above, due to confusing sources near their centers.

For the rest of the targets, no radio emission was detected above $3 \sigma$ that matches the photometric center within the cluster centroid uncertainty or Brownian radius. Figures 2 and 3 show the radio continuum images of these clusters, zoomed in on their centers.

The $3 \sigma$ flux density upper limit was translated to an upper limit on luminosity assuming distances in Tables 1 and 2 and flat radio spectra. The median radio luminosity $3 \sigma$ upper limit is $L_{\mathrm{R}} \lesssim 1.9 \times 10^{27} \mathrm{erg} \mathrm{s}^{-1}$. We then use the formalism described in Section 3.1 to convert this luminosity upper limit to an IMBH mass upper limit. In Figure 7, we plot the IMBH
NGC 6441

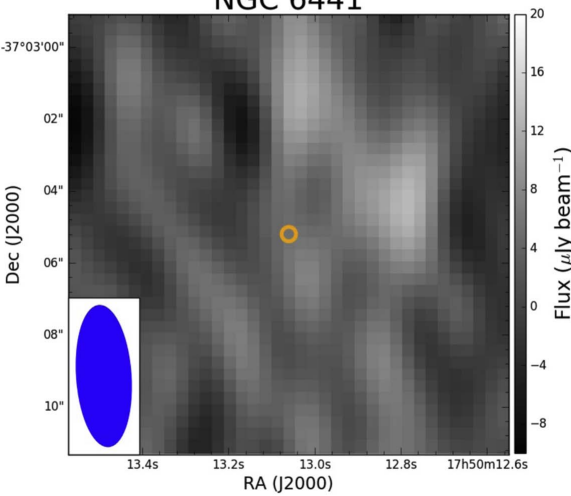

NGC 6541
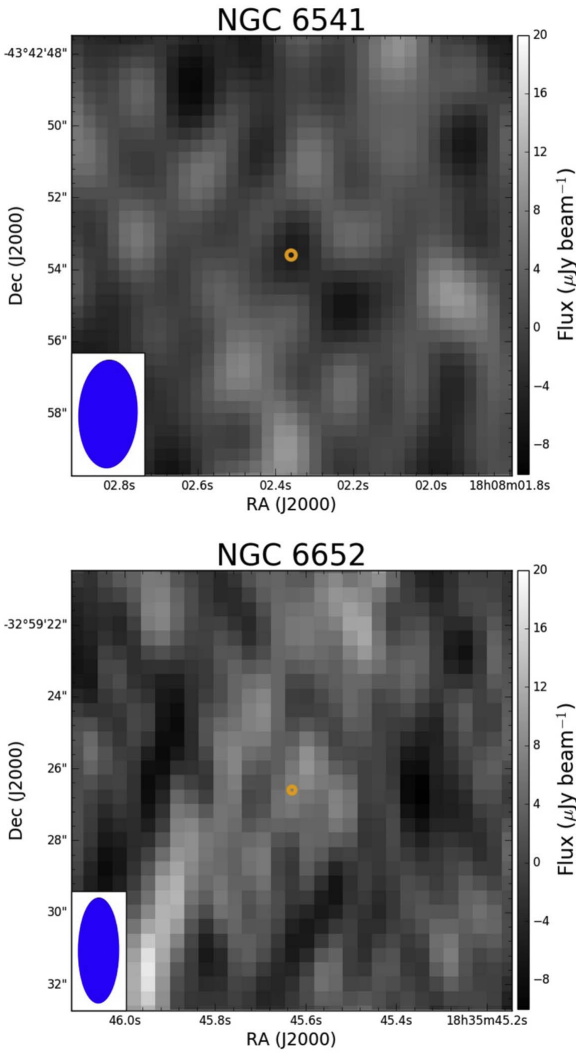

Figure 3. (Continued.)
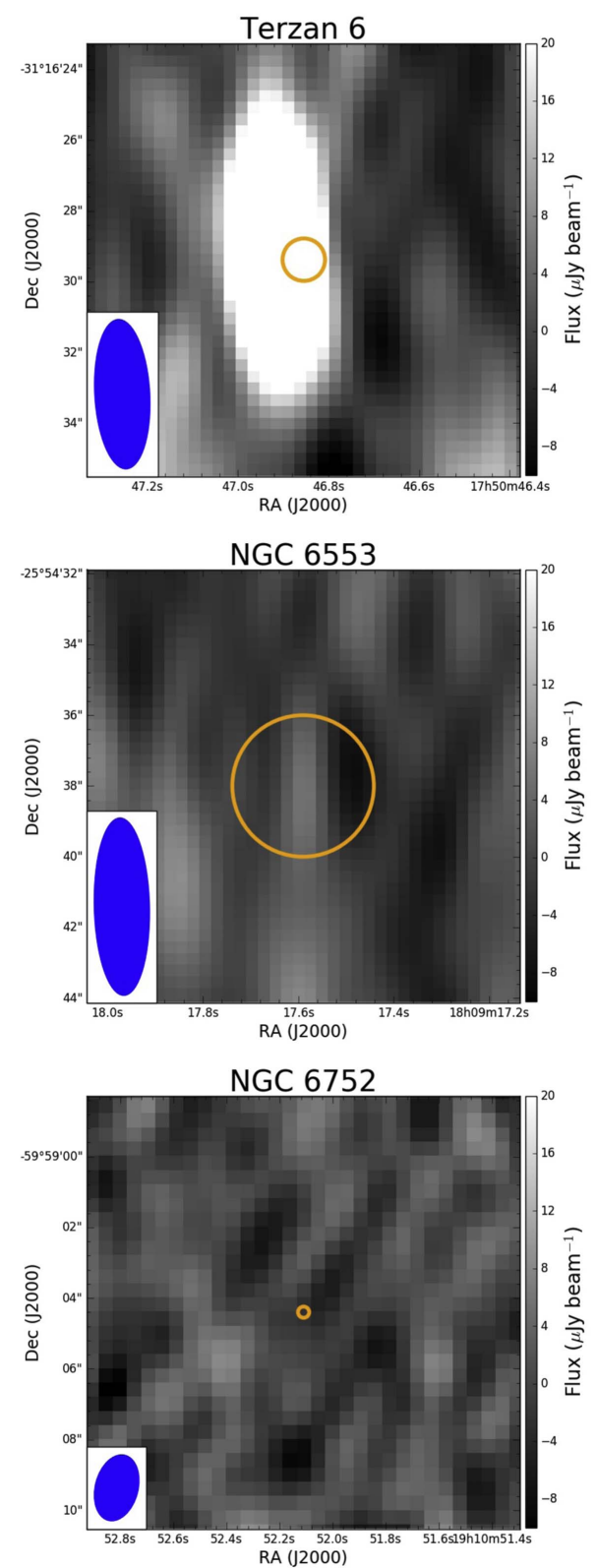


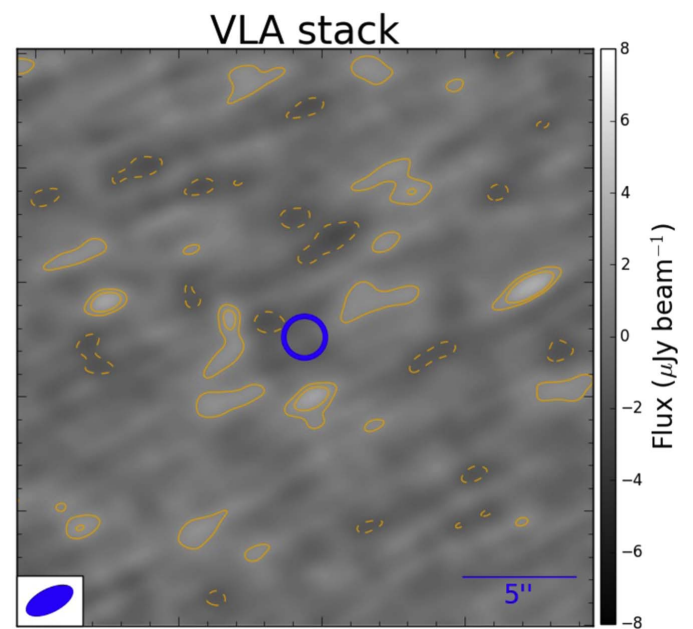

Figure 4. Weighted-mean stack of 24 clusters observed with VLA showing the central 25 !! $2 \times 25$ !! 2 area. The stacked image has an rms noise of $0.65 \mu \mathrm{Jy}$ beam $^{-1}$. The contours represent flux densities of $-2 \sigma$ (dotted), $2 \sigma$, and $3 \sigma$. The blue circle shows the mean IMBH wander radii $(0 . .91)$, and the radio beam is shown in the bottom left corner.

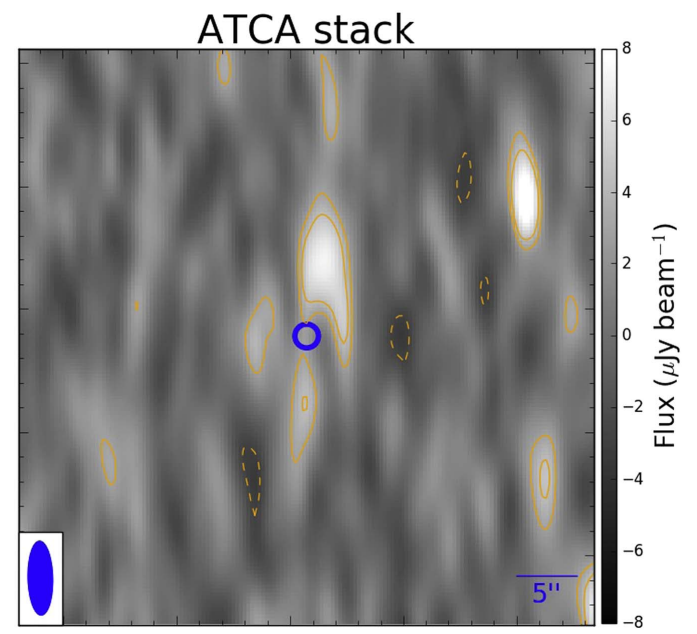

Figure 5. Weighted-mean stack of 14 clusters observed with ATCA showing the central $47 ! .8 \times 47$ !. 8 area. The stacked image has an rms noise of $1.42 \mu \mathrm{Jy}$ beam $^{-1}$. The contours represent flux densities of $-2 \sigma$ (dotted), $2 \sigma$, and $3 \sigma$. The blue circle shows the mean IMBH wander radii $\left(0{ }^{\prime \prime} 96\right)$, and the synthesized beam is shown in the bottom left corner.

These tables also list the $3 \sigma$ IMBH mass limits expressed as a percentage of the total cluster mass. The GC masses are mostly from the recent work of Baumgardt \& Hilker (2018), excepting four GCs not in that paper, for which we use the $M_{V}$ listed in Harris (1996) and assume $M / L_{V}=2$. For the VLA sample, the median $3 \sigma \mathrm{IMBH}$ mass limit is $<0.36 \%$ of the cluster mass; for the ATCA sample, it is $<0.52 \%$. We discuss these limits in the context of theoretical predictions in Section 5.3.

\subsection{Deep Limits from Image Stacking}

The analysis above reflects mass limits on accreting IMBHs in individual GCs. If we instead assume that the IMBH occupation fraction is high, then we can set deeper limits on the presence of such IMBHs by stacking the cluster images.

Section 2.4 describes our technique for co-adding the cluster images, resulting in the deep images presented in Figures 4 and 5. The distant GC M54 and GCs with stellar radio sources coinciding with their photometric centers (Section 3.3) were excluded from these stacks. We also excluded NGC 6139 because there is a $3.2 \sigma$ flux peak 0 " 6 from the cluster center at 9.0 GHz. There is no emission at this location at $5.5 \mathrm{GHz}$, and the $9.0 \mathrm{GHz}$ emission appears to be an artifact associated with a bright source 3!.4 from the center. Djorg 2 does not have a central source but also shows artifacts associated with a bright source 2.5 from the center and is likewise excluded. Finally, NGC 4372 does not contain a significant central source but shows diffuse "fuzz" in the frequency-averaged image. NGC 4372 does not have a particularly large interaction rate (Bahramian et al. 2013), so it is not clear that a population of pulsars is expected; we defer a detailed comparison of interaction rates to radio source populations to a future work and for now exclude NGC 4372 from the ATCA stack.

For the VLA stack we included 24 clusters that have a median (mean) distance of $7.7(6.9) \mathrm{kpc}$. The rms sensitivity of the co-added image is $0.65 \mu \mathrm{Jy}$ beam $^{-1}$, and there is no significant source detected at the center of the stacked image. Using our formalism, this corresponds to a $3 \sigma$ VLA stack limit of $<800 M_{\odot}\left(<730 M_{\odot}\right)$. For this limit, the implied $L_{\mathrm{X}} / L_{\text {edd }} \sim 10^{-11}$.

For the ATCA stacked image we averaged 14 clusters with a median (mean) distance of 6.8 (6.5) kpc. The image rms sensitivity is $1.42 \mu \mathrm{Jy}$ beam $^{-1}$, and no central source is detected. The corresponding $3 \sigma$ ATCA stack limit is $<970 M_{\odot}$.

Since many authors have argued that the densest ("core collapse") clusters are unlikely to contain IMBHs (e.g., Baumgardt et al. 2005; Trenti 2006; Noyola \& Baumgardt 2011), we also created VLA and ATCA stacks excluding those GCs typically identified as core collapsed. Many of these were already excluded for individual reasons as we have noted. As expected, these new stacked images had slightly higher rms values than the full VLA and ATCA stacks (about 1.0 and $1.5 \mu \mathrm{Jy}$ beam $^{-1}$, respectively) and do not show significant central sources. Hence the exclusion of these GCs does not affect any of our conclusions.

\section{Discussion and Conclusions}

Our main result is that there is no accretion evidence for IMBHs with masses $\gtrsim 1000 M_{\odot}$ in any Milky Way GC. We first discuss general uncertainties in our analysis and then specific cases of GCs where IMBHs have been claimed in previous work.

\subsection{Uncertainties on Mass Limits}

The logical basis for our constraints can be divided into three parts: if an IMBH is present, (1) is gas also present, (2) is this gas accreted by the IMBH, and (3) does this accreting gas produce the assumed radio signature?

That some gas is present in the core of GCs is certain-the winds of red giants supply an ongoing flux of material, and ionized gas has been observed in 47 Tuc, with some evidence in M15 (Freire et al. 2001). As 47 Tuc is rich in millisecond pulsars, this obviates the suggestion that such energy sources will reduce the plasma density to negligible amounts (Spergel 1991), though many mechanisms may well be responsible for clearing out much of the gas lost from stars (see, e.g., the discussion in Naiman et al. 2013). Additional 


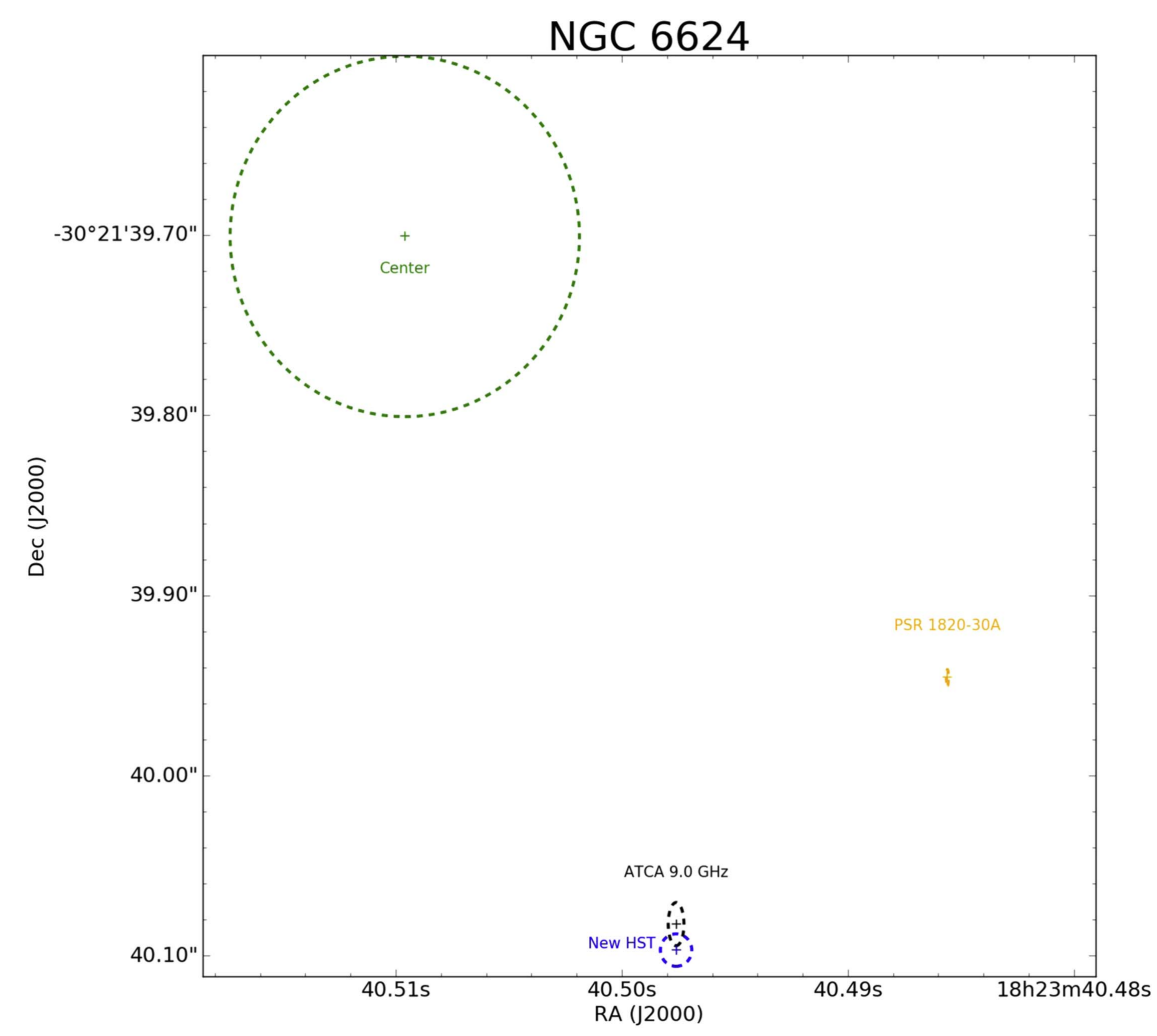

Figure 6. Central region of NGC 6624. The photometric center is marked with a green cross, and the position uncertainty is represented with the green dashed circle ( $\sim 0^{\prime \prime} 1$ in radius). At radio wavelengths, the center of NGC 6624 is dominated by the well-known neutron star sources PSR 1820-30A and 4U 1820-30. The position of the optical counterpart to $4 \mathrm{U} 1820-30$ is shown with the blue dashed circle; the radius denotes the uncertainty on the position. The position and uncertainty of the radio source in our ATCA images is shown as a black ellipse. The position of the PSR 1820-30A is marked in orange (Perera et al. 2017).

measurements of the ionized gas density in clusters and more sophisticated models of the intracluster medium are desirable.

Since no $\sim 1000 M_{\odot}$ IMBHs are known, all discussions of their properties necessarily involve indirect inferences. "Lowmass" central BHs with $\sim 10^{5}-10^{6} M_{\odot}$ are present and are evidently accreting gas in the nuclear star clusters of nearby galaxies (Nguyen et al. 2017; Nyland et al. 2017), with even lower-mass BHs detected at higher accretion rates in more distant galaxies (Baldassare et al. 2015). At least in terms of their mass, these systems probably represent the nearest analogues to IMBHs in GCs, and nothing prevents accretion onto the central $\mathrm{BH}$. We have also assumed that the radiative efficiency and accretion rate, parameterized in terms of the Bondi rate, have typical values comparable to nearby lowluminosity accreting central BHs. These observed systems have a large dispersion in the accretion rate and/or efficiency. Some may not have appreciable accretion at all, though others could be accreting at much higher rates or with higher efficiency than assumed. If the dispersion in these quantities is high, then the nondetection of IMBHs in 50 GCs strongly suggests that IMBHs are rare, if they exist at all. We emphasize that the actual accretion rates and radiative efficiencies of IMBHs are the chief uncertainty in this analysis.

Depending on the assumptions for radiative efficiency, our typical VLA limits correspond to very low accretion rates of a few $\times 10^{-11} M_{\odot} \mathrm{yr}^{-1}$, which is only a few percent of the mass loss rate of one red giant (Dupree et al. 2009). We note that MacLeod et al. (2016) argued that some fraction of GC IMBHs should have nondegenerate companions that could supply a higher rate of gas to the IMBH than accretion from ambient material.

The use of the fundamental plane to convert radio limits to masses is an interpolation between stellar-mass and supermassive BHs rather than an extrapolation. This fact is heartening, but the accretion behavior of hypothetical IMBHs is still unknown. Cseh et al. (2015) found that HLX-1 was more radio bright than predicted by the fundamental plane, assuming that the mass inferred from X-ray spectral fitting was correct. The large (0.4 dex; Plotkin et al. 2012) scatter in the fundamental plane also limits precise statements about accretion implications for specific systems.

\subsection{Revisiting IMBHs from the Literature}

We begin with $\omega$ Cen and M54, as these massive GCs are often argued to be stripped galaxy nuclei (the case for M54 is especially strong; Layden \& Sarajedini 2000) and hence may be the most likely to host IMBHs.

$$
\text { 5.2.1. } \omega \text { Cen }
$$

$\omega$ Cen has many contrasting claims of dynamical evidence for an IMBH (Noyola et al. 2008; Noyola 2010; van der Marel \& Anderson 2010; Baumgardt 2017), with some papers finding dynamical evidence for an IMBH with a mass of $\gtrsim 10^{4} M_{\odot}$. We do not revisit this work here but focus solely on the accretion constraints. Our $3 \sigma$ ATCA upper limit of $<8.9 \mu \mathrm{Jy}$ on a central radio source implies a $3 \sigma$ IMBH mass upper limit of $<1000 M_{\odot}$ using our formalism. The corresponding $0.5-10 \mathrm{keV}$ $\mathrm{X}$-ray luminosity limit is $<2.5 \times 10^{30} \mathrm{erg} \mathrm{s}^{-1}$ (Table 2), which 
can be compared to the observed (95\%) upper limit of $<1.7 \times 10^{30} \mathrm{erg} \mathrm{s}^{-1}$ (Haggard et al. 2013). Because of the large amount of Chandra data on this cluster, this is one case where the X-ray limit is as (or more) constraining than the radio limit. As discussed in Haggard et al. (2013) and Strader et al. (2012), if an IMBH with a mass of $\gtrsim 10^{4} M_{\odot}$ is present in $\omega$ Cen, then it must be accreting at a relative rate below any other central BH known in the universe, with $L_{\text {bol }} / L_{\text {edd }} \lesssim 2 \times 10^{-11}$. There is no accretion evidence for an IMBH in $\omega$ Cen.

\subsection{2. $M 54$}

In M54, the $3 \sigma$ radio upper limit of $<7.2 \mu \mathrm{Jy}$ gives a mass limit of $<3000 M_{\odot}$, far below the $9400 M_{\odot}$ suggested dynamically (Ibata et al. 2009). The new radio limit is about a factor of seven stronger than the one presented in Wrobel et al. (2011), due entirely to the improved sensitivity of the postupgrade VLA. M54 is the nucleus of the Sagittarius dwarf galaxy (e.g., Monaco et al. 2005) with a $V$ band stellar luminosity of $10^{8} L_{\odot}$ and a halo mass of $>6 \times 10^{10} M_{\odot}$ (Gibbons et al. 2017). While the occupation fraction of BHs is known to be high at the centers of slightly higher-mass galaxies (Nguyen et al. 2017), little is known about the BH occupation fraction at these low masses. Owing to its identity as the closest confirmed galaxy nucleus beyond Sgr A*, in our view M54 presents a strong case for even deeper radio observations.

\subsubsection{New Pulsar Evidence: 47 Tuc and NGC 6624}

For many years, 47 Tuc was a rare case where most papers agreed that there was no substantial evidence for an IMBH; most prominently, McLaughlin et al. (2006) set a $1 \sigma$ dynamical upper limit of $<1000-1500 M_{\odot}$ on an IMBH. This was challenged by Kiziltan et al. (2017), who used the timing of millisecond pulsars in the core of 47 Tuc to argue for the presence of a $2300 M_{\odot}$ IMBH. Freire et al. (2017) disputed this interpretation of the observations (partially on the basis of the assumed cluster distance) and argued that no IMBH is necessary to explain the pulsar timing data. Our new ATCA $3 \sigma$ radio upper limit of $<11.2 \mu \mathrm{Jy}$ corresponds to a mass limit of $<1040 M_{\odot}$, suggesting that an IMBH of the mass published by Kiziltan et al. (2017) is not present or that it is accreting at a rate or efficiency lower than assumed in our formalism. 47 Tuc is another cluster, like $\omega$ Cen, where there are extremely deep Chandra data (Grindlay et al. 2001), which limit a central X-ray source to $<10^{31} \mathrm{erg} \mathrm{s}^{-1}(0.5-10 \mathrm{keV})$. The corresponding $3 \sigma$ limit from our formalism is $L_{\mathrm{X}}<2.8 \times$ $10^{30} \mathrm{erg} \mathrm{s}^{-\mathrm{P}}$, and a deeper Chandra constraint should be possible in the future through the analysis of archival data.

A unique recent claim of dynamical evidence for an IMBH in the GC NGC 6624 comes from Perera et al. (2017), who argued, on the basis of precise long-term timing, that the pulsar PSR 1820-30A is in a wide eccentric orbit around an IMBH. The observational interpretation of this cluster is complicated, and we discuss it in detail in the Appendix. Here we only mention our ATCA radio limit on a central IMBH: a $3 \sigma$ value of $<9.8 \mu \mathrm{Jy}$, giving a $3 \sigma \mathrm{IMBH}$ mass of $<1550 M_{\odot}$, compared to a dynamical IMBH mass of $7500-10,000 M_{\odot}$ in Perera et al. (2017). With our formalism, the radio flux density of a $7500 M_{\odot}$ IMBH would be predicted to be about $700 \mu \mathrm{Jy}$, which is about a factor of 70 larger than our ATCA limit.

\subsubsection{Other Clusters}

NGC 6388 is a case similar to $\omega$ Cen where there is disagreement in the literature about dynamical evidence for the presence of a (2-3) $\times 10^{4} M_{\odot}$ IMBH (Lützgendorf et al. 2011; Lanzoni et al. 2013; Lützgendorf et al. 2015). Previous ATCA observations gave $3 \sigma$ upper limits of $<81 \mu \mathrm{Jy}$ (at $9 \mathrm{GHz}$; Cseh et al. 2010) and $<42 \mu \mathrm{Jy}$ (at $5.5 \mathrm{GHz}$; Bozzo et al. 2011). As discussed above, we do observe emission near (but not coincident with) the center of the cluster at $5.5 \mathrm{GHz}$, which we attribute to unresolved pulsars. Such emission is not unexpected given the strong Fermi GeV flux from the cluster. At $9.0 \mathrm{GHz}$ no emission is detected, with a $3 \sigma$ upper limit of $<8.5 \mu \mathrm{Jy}$, about a factor of five lower than the Bozzo et al. (2011) limit. This corresponds to a $3 \sigma$ IMBH mass limit of $<1770 M_{\odot}$, about a factor of 16 lower than the dynamical mass inferred from Lützgendorf et al. (2015).

M15 is a cluster where there were early dynamical hints for an IMBH (Newell 1970; Gerssen et al. 2002, 2003), for which the interpretation was immediately disputed (Illingworth \& King 1977; Baumgardt et al. 2003), and subsequent works have generally agreed that no IMBH is required (e.g. den Brok et al. 2014) . Our M15 radio data are the same as presented in Strader et al. (2012), which found no accretion evidence for an IMBH.

There are other clusters for which single studies have suggested dynamical evidence for IMBHs. Lützgendorf et al. (2013a) presented $2 \sigma$ dynamical evidence for a $\sim 2000 M_{\odot}$ IMBH in M62, for which our formal VLA $3 \sigma$ limit is $<1130 M_{\odot}$. Kamann et al. (2016) suggested that NGC 6397 could host a $600 M_{\odot}$ IMBH. This does not conflict with our ATCA limit for this cluster $\left(<630 M_{\odot}\right)$. For both of these clusters, Baumgardt (2017) argued that the surface brightness profiles and kinematic data do not require the presence of an IMBH.

\subsection{Conclusions and Implications}

We have presented the deepest radio observations to date for a sample of 50 Milky Way GCs, with a goal of searching for evidence of central accreting IMBHs. While a few clusters do have radio sources near or in their centers, we do not find any credible IMBH candidates. A stacking analysis of GCs observed with VLA or ATCA also reveals no emission that can be attributed to IMBHs.

We emphasize that for any particular GC it is possible to conceive of mechanisms that would reduce or eliminate the accretion of ambient gas, rendering the IMBH undetectable in current radio or X-ray observations. Yet it is difficult to argue that such conditions should apply in many or most GCs. The most straightforward conclusion to draw from our work is that IMBHs with masses $\gtrsim 10^{3} M_{\odot}$ are either not present or at least not common in GCs.

The recent detections of merging binary BHs through gravitational waves (Abbott et al. 2016) may help explain the observed lack of IMBHs in GCs. If such binary stellar-mass BHs are formed dynamically in GCs (Rodriguez et al. 2016), then it could indicate that single and binary BHs are preferentially ejected from GCs rather than merging with a more massive seed $\mathrm{BH}$ to form an IMBH (Miller \& Hamilton 2002; Gültekin et al. 2004, 2006; Baker et al. 2008; Moody \& Sigurdsson 2009). Of course, there are many ways to grow IMBHs in GCs that do not depend on mergers of smaller BHs (e.g., Portegies Zwart et al. 2004). 
We also cannot constrain IMBHs that may have been ejected from GCs (Holley-Bockelmann et al. 2008) or the presence of less massive IMBHs (those of a few hundred $M_{\odot}$ ) through accretion signatures, especially since such objects may wander far from the cluster center (Lützgendorf et al. 2013b).Theoretical predictions for IMBH masses range widely, from $0.1 \%$ to $1 \%$ or more of the cluster mass (e.g., Miller \& Hamilton 2002; O'Leary et al. 2006; Portegies Zwart et al. 2006; Giersz et al. 2015; Woods et al. 2017). Our median VLA and ATCA limits are in the middle of this range $(0.36 \%$ and $0.54 \%)$, though the extreme values range as low as $0.03 \%$ and as high as $2.3 \%$. If IMBHs typically make up only $0.1 \%$ of the mass of a GC, then (in the context of our formalism for radio emission) they would be difficult to detect outside the most massive GCs, unless the accretion is more radiatively efficient than we assume. We note that IMBHs well below $1000 M_{\odot}$ are also not easily detected via standard dynamical techniques (Baumgardt 2017). Future gravitational wave observatories, including eLISA, offer more hope for detecting such IMBHs (Haster et al. 2016) —if they exist.

We thank an anonymous referee and B. Stappers and B. Perera for useful comments. The National Radio Astronomy Observatory is a facility of the National Science Foundation (NSF) operated under cooperative agreement by Associated Universities, Inc. The ATCA is part of the Australia Telescope National Facility, which is funded by the Australian government for operation as a national facility managed by CSIRO.

J.S. acknowledges support from the Packard Foundation. We thank the NSF through support from grants AST-1308124 and AST-1514763. J.C.A.M.-J. is the recipient of an Australian Research Council Future Fellowship (FT140101082). C.O.H. and G.R.S. are supported by NSERC Discovery Grants, and C.O.H. is also supported by an NSERC Discovery Accelerator Supplement. A.C.S. acknowledges financial support from NSF AST-1350389.

Facilities: VLA, ATCA.

Software: CASA (McMullin et al. 2007), AIPS (Wells 1985), TOPCAT (Taylor 2005), APLpy (Robitaille \& Bressert 2012), Astropy (Astropy Collaboration et al. 2013).

\section{Appendix}

\section{A.1. NGC 6624: The Position of $4 U$ 1820-30}

A continuing discussion about the radio continuum imaging of NGC 6624 is whether central radio emission can be attributed to the low-mass X-ray binary 4U 1820-30, the bright pulsar PSR 1820-30A, or a frequency-dependent combination of the two. Owing to the steep spectral slope of the pulsar, it may significantly contribute at low frequencies, but at the higher frequencies of our ATCA observations the contribution of the pulsar is expected to be minimal (Migliari et al. 2004). One issue with this argument in the past has been that the location of the radio emission was not entirely consistent with that of $4 \mathrm{U}$ 1820-30, independently determined via $H S T$ imaging of the UV-bright optical counterpart to the X-ray binary.

We first revisit the position of the optical counterpart to $4 \mathrm{U}$ 1820-30 using new HST data taken with WFC3 (Program GO13297; P.I. Piotto). 4U 1820-30 is the brightest source in the core of the cluster in F275W; however, many of the Gaia stars in the initial DR1 data release in the field of view of this image are not well detected. Hence we instead focus on F336W, in which $4 \mathrm{U}$
1820-30 is still bright but the number of well-detected Gaia stars is larger. We correct the astrometry of the F336W HST image using the Gaia stars, achieving a solution with an rms uncertainty of 13-14 mas per coordinate. The J2000 position of this star is (R.A., decl.) $=(18: 23: 40.4975 \pm 0.0010$ s, 30:21:40.096 \pm 0 ". 017).

To determine the position of the radio source in our ATCA images we use the $9.0 \mathrm{GHz}$ image, which has the best resolution, finding a $\mathrm{J} 2000$ position of (R.A., decl.) = $(18: 23: 40.4978 \pm 0.0005 s,-30: 21: 40.081 \pm 0$ ". 024). The difference between our ATCA $9.0 \mathrm{GHz}$ position and the $H S T$ $F 336 \mathrm{~W}$ position is $0 . " 016$ - the two positions agree even within their small uncertainties. Hence we conclude that the radio emission at $9.0 \mathrm{GHz}$ is entirely due to $4 \mathrm{U} 1820-30$ and that the position of this binary is well determined. $4 \mathrm{U} 1820-30$ is located 0 " 43 from the cluster center.

We next compare this position to that found in previous work. It is just outside of the larger error circle of the VLA source found by Migliari et al. (2004) at 4.9 and $8.4 \mathrm{GHz}-$ consistent at the $1.3 \sigma$ level. However, it is entirely inconsistent with the previous HST position by Sosin \& King (1995), differing by $0 . " 58$. This has important implications for the interpretation of the properties of $4 \mathrm{U}$ 1820-30: rather than being more distant from the cluster center than PSR 1820-30A, it is at a similar distance.

We note that our new position for 4U 1820-30 differs from the ALMA position found by Díaz Trigo et al. (2017) by 0". 36; this difference is nominally highly significant given the stated positional uncertainties. However, both positions are approximately the same distance from the cluster center (about 0 ". 4 in both cases), so they do not change the interpretation of the X-ray binary properties in the context of an IMBH. Understanding the source of this discrepancy is beyond the scope of the current paper.

\section{A.2. Implications}

Perera et al. (2017) studied long-term radio timing observations of PSR 1820-30A, located close to the center of NGC 6624. These observations admit two possibilities: that PSR $1820-30 \mathrm{~A}$ is in an extremely wide, eccentric orbit around a massive IMBH, or that it is in a less-eccentric orbit with a normal star or stellar remnant. One of their arguments in favor of the IMBH interpretation is that the unexpected negative orbital period derivative of $4 \mathrm{U} 1820-30$ is due to an acceleration that requires the addition of an IMBH to the cluster potential.

From reviewing the literature it is clear that a series of errors has propagated since the earliest identification of 4U 1820-30 with HST. The first paper to locate the source was King et al. (1993), who found that 4U 1820-30 was located 0". $66 \mathrm{~N}$ of the cluster center (from their abstract; the listed positions actually imply only $0 . " 6$, possibly due to rounding). Sosin \& King (1995) "corrected" a 1". 8 error in the absolute astrometry of King et al. (1993) and refined the cluster center, but the relative distance of $4 \mathrm{U}$ 1820-30 from the center was essentially unchanged at 0 .' 67.

Peuten et al. (2014) reported that if the updated Goldsbury et al. (2010) center is used, then $4 \mathrm{U} 1820-30$ is $0.046 \mathrm{pc}$ from the cluster center (1".2 at Peuten et al. 2014's assumed distance of $7.9 \mathrm{kpc}$ ). We are unable to determine the origin of this value. The distance of the King et al. (1993) position from the Goldsbury et al. (2010) center is 0."98; if the X-ray binary 


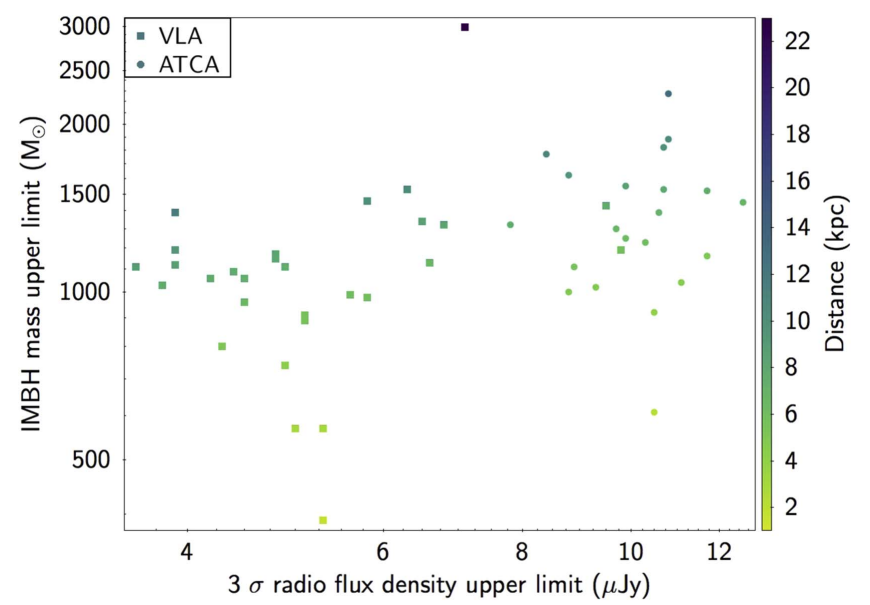

Figure 7. IMBH mass upper limits as a function of radio flux density upper limits for VLA (squares) and ATCA (circles). The colors of symbols denote distance, as shown in the color-bar at right. Both axes are plotted in logarithmic scales. M4 shows the lowest IMBH mass upper limit while the next-lowest IMBH upper limits from the VLA are for NGC 6544 and M22. From ATCA data, NGC 6397 has the lowest IMBH mass upper limit.

position from Sosin \& King (1995) is used, then the distance from the new center is 0 ". 83 .

Perera et al. (2017) cited Migliari et al. (2004) for the position of $4 \mathrm{U}$ 1820-30, who in turn took the position from Sosin \& King (1995). However, the value published by Migliari et al. (2004) is rounded off, and hence the separation of $4 \mathrm{U} 1820-30$ from the center implied is slightly larger than the correct value $(0$ ". 87 instead of 0 ". 83$)$. Notwithstanding this rounding, the positions in their Table 1 and Figure 1 are accurately rendered and would imply a separation of $0.032 \mathrm{pc}$ (for $7.9 \mathrm{kpc}$ ). However, in their Figure 9 that summarizes the dynamical constraints, and in their Erratum Figure 1, the separation plotted for $4 \mathrm{U} 1820-30$ is simply the physical value from Peuten et al. (2014), 0.046 pc. The incorrect separation from Peuten et al. (2014) is repeated in Gieles et al. (2018), who argued, contra Perera et al. (2017), that in any case most or all of the period change can be attributed to intrinsic factors.

The propagation of this mistake through the literature caused an overinterpretation of the $4 \mathrm{U} 1820-30$ period derivative when only considering the information available. Our new HST position doubles down: we find that $4 \mathrm{U} 1820-30$ is $0.43 \pm$ 0 " 10 from the Goldsbury et al. (2010) center. This is equivalent to $0.0175 \pm 0.0041 \mathrm{pc}$ using our assumed distance of $8.4 \mathrm{kpc}$ (using $7.9 \mathrm{kpc}$ instead would not change any of the conclusions). This is nearly identical to the separation between PSR 1820-30A and the center using the updated position of the pulsar from Perera et al. (2017); given the uncertainties in the center, either source may actually be closer. We do not present updated dynamical models of NGC 6624 in this paper, but only note that using the correct position of $4 \mathrm{U} 1820-30$ would lower the inferred IMBH mass - if its period derivative is interpreted as being dominated by the potential of an IMBH, rather than intrinsic factors.

\section{A.3. The Radio Properties of $4 U$ 1820-30}

Considering the radio properties of $4 \mathrm{U}$ 1820-30 itself: the flux density of $4 \mathrm{U} 1820-30$ is $235 \pm 4 \mu \mathrm{Jy}(5.5 \mathrm{GHz})$ and $207 \pm 4 \mu \mathrm{Jy}(9.0 \mathrm{GHz})$, yielding a spectral index of $\alpha=-0.26 \pm 0.06$ for a power-law $S_{\nu} \propto \nu^{\alpha}$. Previous radio continuum observations at similar frequencies have been made with the VLA (mean flux densities of $130 \pm 40$ and $100 \pm 20 \mu \mathrm{Jy}$ at 4.9 and $8.4 \mathrm{GHz}$, respectively; Migliari et al. 2004) and with ATCA (flux densities of $236 \pm 27$ and $<200 \mu \mathrm{Jy}$ at 5.5 and 9.0 GHz; Díaz Trigo et al. 2017). Our new flux densities are a factor of $\sim 2$ higher than those measured with the old VLA, possibly related to the well-known superorbital modulation in X-rays (Chou \& Grindlay 2001). Our $5.5 \mathrm{GHz}$ ATCA measurement is spot on with the 2014 ATCA measurement made simultaneously with ALMA observations (Díaz Trigo et al. 2017). Our 9.0 GHz measurement is nominally inconsistent with the upper limit of $<200 \mu \mathrm{Jy}$ reported by Díaz Trigo et al. (2017), but only marginally so. In any case, the flux density of 4U 1820-30 and its spectral slope are extremely well measured in these new observations.

Given that we find a flux density at $5.5 \mathrm{GHz}$ identical to Díaz Trigo et al. (2017), it seems reasonable to combine their 302 $\mathrm{GHz}$ ALMA flux density of $400 \pm 20 \mu \mathrm{Jy}$ with our ATCA measurements at 5.5 and $9.0 \mathrm{GHz}$ to determine the radio $/ \mathrm{mm}$ spectral energy distribution of the binary. In this case, we find that the 5.5, 9.0 and $302 \mathrm{GHz}$ flux densities are strongly inconsistent with a single power law. This suggests either that the ALMA observations (taken 1 week from the 2014 ATCA data) were taken during a flare unobserved at other wavelengths or that there is another source of $302 \mathrm{GHz}$ emission. Truly simultaneous radio and mm observations of 4U 1820-30 appear necessary to determine an accurate spectral energy distribution for this binary.

\section{ORCID iDs}

Evangelia Tremou (i) https://orcid.org/0000-0002-4039-6703 Jay Strader (i) https://orcid.org/0000-0002-1468-9668 Laura Chomiuk (iD https://orcid.org/0000-0002-8400-3705 James C. A. Miller-Jones (i) https://orcid.org/0000-00033124-2814

Craig O. Heinke (iD https://orcid.org/0000-0003-3944-6109 Gregory R. Sivakoff (iD https://orcid.org/0000-00016682-916X

\section{References}

Abbott, B. P., Abbott, R., Abbott, T. D., et al. 2016, PhRvL, 116, 061102 Abdo, A. A., Ackermann, M., Ajello, M., et al. 2010, A\&A, 524, A75 Anderson, J., \& van der Marel, R. P. 2010, ApJ, 710, 1032

Arellano Ferro, A., Bramich, D. M., Figuera Jaimes, R., et al. 2013, MNRAS, 434, 1220

Astropy Collaboration, Robitaille, T. P., Tollerud, E. J., et al. 2013, A\&A, 558, A33

Bahramian, A., Heinke, C. O., Sivakoff, G. R., \& Gladstone, J. C. 2013, ApJ, 766,136

Baker, J. G., Boggs, W. D., Centrella, J., et al. 2008, ApJL, 682, L29

Baldassare, V. F., Reines, A. E., Gallo, E., \& Greene, J. E. 2015, ApJL, 809, L14

Baumgardt, H. 2017, MNRAS, 464, 2174

Baumgardt, H., \& Hilker, M. 2018, MNRAS, 478, 1520

Baumgardt, H., Hut, P., Makino, J., McMillan, S., \& Portegies Zwart, S. 2003, ApJL, 582, L21

Baumgardt, H., Makino, J., \& Hut, P. 2005, ApJ, 620, 238

Blandford, R. D., \& Königl, A. 1979, ApJ, 232, 34

Bogdanov, S., Heinke, C. O., Özel, F., \& Güver, T. 2016, ApJ, 831, 184

Bondi, H. 1952, MNRAS, 112, 195

Bozzo, E., Ferrigno, C., Stevens, J., et al. 2011, A\&A, 535, L1

Bromm, V., \& Loeb, A. 2003, ApJ, 596, 34

Cackett, E. M., Wijnands, R., Heinke, C. O., et al. 2006, MNRAS, 369, 407

Cadelano, M., Ransom, S. M., Freire, P. C. C., et al. 2018, ApJ, 855, 125

Chatterjee, P., Hernquist, L., \& Loeb, A. 2002, ApJ, 572, 371 
Chou, Y., \& Grindlay, J. E. 2001, ApJ, 563, 934

Cohen, J. G., Hsieh, S., Metchev, S., Djorgovski, S. G., \& Malkan, M. 2007, AJ, 133, 99

Cohen, R. E., Mauro, F., Geisler, D., et al. 2014, AJ, 148, 18

Contreras, R., Catelan, M., Smith, H. A., et al. 2010, AJ, 140, 1766

Cseh, D., Kaaret, P., Corbel, S., et al. 2010, MNRAS, 406, 1049

Cseh, D., Webb, N. A., Godet, O., et al. 2015, MNRAS, 446, 3268

Dalessandro, E., Beccari, G., Lanzoni, B., et al. 2009, ApJS, 182, 509

Dalessandro, E., Lanzoni, B., Beccari, G., et al. 2011, ApJ, 743, 11

D’Amico, N., Lyne, A. G., Manchester, R. N., Possenti, A., \& Camilo, F. 2001, ApJL, 548, L171

de Vita, R., Trenti, M., \& MacLeod, M. 2018, MNRAS, 475, 1574

DeCesar, M. E., Ransom, S. M., Kaplan, D. L., Ray, P. S., \& Geller, A. M. 2015, ApJL, 807, L23

den Brok, M., van de Ven, G., van den Bosch, R., \& Watkins, L. 2014, MNRAS, 438, 487

Díaz Trigo, M., Migliari, S., Miller-Jones, J. C. A., et al. 2017, A\&A, 600, A8 Djorgovski, S. 1987, ApJL, 317, L13

Dupree, A. K., Smith, G. H., \& Strader, J. 2009, AJ, 138, 1485

Eisenstein, D. J., \& Loeb, A. 1995, ApJ, 443, 11

Falcke, H., Körding, E., \& Markoff, S. 2004, A\&A, 414, 895

Fall, S. M., \& Rees, M. J. 1985, ApJ, 298, 18

Farrell, S. A., Servillat, M., Gladstone, J. C., et al. 2014, MNRAS, 437, 1208

Feldmeier, A., Lützgendorf, N., Neumayer, N., et al. 2013, A\&A, 554, A63

Feng, H., \& Soria, R. 2011, NewAR, 55, 166

Ferrara, A., Haardt, F., \& Salvaterra, R. 2013, MNRAS, 434, 2600

Ferraro, F. R., Messineo, M., Fusi Pecci, F., et al. 1999, AJ, 118, 1738

Fregeau, J. M., Joshi, K. J., Portegies Zwart, S. F., et al. 2002, ApJ, 570, 171

Freire, P. C., Kramer, M., Lyne, A. G., et al. 2001, ApJL, 557, L105

Freire, P. C. C., Ridolfi, A., Kramer, M., et al. 2017, MNRAS, 471, 857

Fruchter, A. S., \& Goss, W. M. 2000, ApJ, 536, 865

Gallo, E., Fender, R. P., \& Hynes, R. I. 2005, MNRAS, 356, 1017

Gebhardt, K., Rich, R. M., \& Ho, L. C. 2005, ApJ, 634, 1093

Gerssen, J., van der Marel, R. P., Gebhardt, K., et al. 2002, AJ, 124, 3270

Gerssen, J., van der Marel, R. P., Gebhardt, K., et al. 2003, AJ, 125, 376

Gibbons, S. L. J., Belokurov, V., \& Evans, N. W. 2017, MNRAS, 464, 794

Gieles, M., Balbinot, E., Yaaqib, R. I. S. M., et al. 2018, MNRAS, 473, 4832

Giersz, M., Leigh, N., Hypki, A., et al. 2015, MNRAS, 454, 3150

Goldsbury, R., Richer, H. B., Anderson, J., et al. 2010, AJ, 140, 1830

Greisen, E. W. 2003, ASSL, 285, 109

Grindlay, J. E., Heinke, C., Edmonds, P. D., et al. 2001, Sci, 292, 2290

Gültekin, K., Miller, M. C., \& Hamilton, D. P. 2004, ApJ, 616, 221

Gültekin, K., Miller, M. C., \& Hamilton, D. P. 2006, ApJ, 640, 156

Gürkan, M. A., Freitag, M., \& Rasio, F. A. 2004, ApJ, 604, 632

Haggard, D., Cool, A. M., Heinke, C. O., et al. 2013, ApJL, 773, L31

Harris, W. E. 1996, AJ, 112, 1487

Harris, W. E. 2010, arXiv:1012.3224

Haster, C.-J., Antonini, F., Kalogera, V., \& Mandel, I. 2016, ApJ, 832, 192

Hjellming, R. M., \& Johnston, K. J. 1988, ApJ, 328, 600

Holley-Bockelmann, K., Gültekin, K., Shoemaker, D., \& Yunes, N. 2008, ApJ, 686,829

Ibata, R., Bellazzini, M., Chapman, S. C., et al. 2009, ApJL, 699, L169

Illingworth, G., \& King, I. R. 1977, ApJL, 218, L109

in't Zand, J. J. M., Hulleman, F., Markwardt, C. B., et al. 2003, A\&A, 406, 233

Inayoshi, K., Ostriker, J. P., Haiman, Z., \& Kuiper, R. 2018, MNRAS, 476, 1412

Johnston, H. M., Kulkarni, S. R., \& Goss, W. M. 1991, ApJL, 382, L89

Kaluzny, J., Thompson, I. B., Rozyczka, M., \& Krzeminski, W. 2013, AcA, 63, 181

Kamann, S., Husser, T.-O., Brinchmann, J., et al. 2016, A\&A, 588, A149

Kamann, S., Wisotzki, L., Roth, M. M., et al. 2014, A\&A, 566, A58

King, I. R., Stanford, S. A., Albrecht, R., et al. 1993, ApJL, 413, L117

Kiziltan, B., Baumgardt, H., \& Loeb, A. 2017, Natur, 542, 203

Kunder, A., Stetson, P. B., Cassisi, S., et al. 2013, AJ, 146, 119

Lanzoni, B., Ferraro, F. R., Dalessandro, E., et al. 2010, ApJ, 717, 653

Lanzoni, B., Mucciarelli, A., Origlia, L., et al. 2013, ApJ, 769, 107

Layden, A. C., \& Sarajedini, A. 2000, AJ, 119, 1760

Lindroos, L., Knudsen, K. K., Vlemmings, W., Conway, J., \& Martí-Vidal, I. 2015, MNRAS, 446, 3502

Lodato, G., \& Natarajan, P. 2006, MNRAS, 371, 1813

Loeb, A., \& Rasio, F. A. 1994, ApJ, 432, 52

Lu, T.-N., \& Kong, A. K. H. 2011, ApJL, 729, L25

Lützgendorf, N., Gebhardt, K., Baumgardt, H., et al. 2015, A\&A, 581, A1

Lützgendorf, N., Kissler-Patig, M., Gebhardt, K., et al. 2013a, A\&A, 552, A49

Lützgendorf, N., Kissler-Patig, M., Neumayer, N., et al. 2013b, A\&A, $555, \mathrm{~A} 26$
Lützgendorf, N., Kissler-Patig, M., Noyola, E., et al. 2011, A\&A, 533, A36 Lynch, R. S., Freire, P. C. C., Ransom, S. M., \& Jacoby, B. A. 2012, ApJ, 745, 109

Maccarone, T. J. 2003, A\&A, 409, 697

Maccarone, T. J. 2004, MNRAS, 351, 1049

Maccarone, T. J., Fender, R. P., \& Tzioumis, A. K. 2005, Ap\&SS, 300, 239

Maccarone, T. J., \& Servillat, M. 2008, MNRAS, 389, 379

MacLeod, M., Trenti, M., \& Ramirez-Ruiz, E. 2016, ApJ, 819, 70

Madau, P., \& Rees, M. J. 2001, ApJL, 551, L27

Matsubayashi, T., Makino, J., \& Ebisuzaki, T. 2007, ApJ, 656, 879

Maxwell, J. E., Lugger, P. M., Cohn, H. N., et al. 2012, ApJ, 756, 147

McLaughlin, D. E., Anderson, J., Meylan, G., et al. 2006, ApJS, 166, 249

McMullin, J. P., Waters, B., Schiebel, D., Young, W., \& Golap, K. 2007, in

ASP Conf. Ser. 376, adass XVI, ed. R. A. Shaw, F. Hill, \& D. J. Bell (San Francisco, CA: ASP), 127

Merloni, A., Heinz, S., \& di Matteo, T. 2003, MNRAS, 345, 1057

Migliari, S., Fender, R. P., Rupen, M., et al. 2004, MNRAS, 351, 186

Miller, M. C., \& Hamilton, D. P. 2002, MNRAS, 330, 232

Miller-Jones, J. C. A., Sivakoff, G. R., Heinke, C. O., et al. 2011, ATel, 3378

Miller-Jones, J. C. A., Wrobel, J. M., Sivakoff, G. R., et al. 2012, ApJL, 755, L1

Miocchi, P., Lanzoni, B., Ferraro, F. R., et al. 2013, ApJ, 774, 151

Monaco, L., Bellazzini, M., Ferraro, F. R., \& Pancino, E. 2005, MNRAS, 356,1396

Moody, K., \& Sigurdsson, S. 2009, ApJ, 690, 1370

Musaeva, A., Koribalski, B. S., Farrell, S. A., et al. 2015, MNRAS, 447, 1951

Nagar, N. M., Falcke, H., Wilson, A. S., et al. 2000, ApJ, 542, 186

Naiman, J., Soares-Furtado, M., \& Ramirez-Ruiz, E. 2013, ApJ, submitted (arXiv:1310.8301)

Narayan, R., \& Yi, I. 1995, ApJ, 452, 710

Newell, B., da Costa, G. S., \& Norris, J. 1976, ApJL, 208, L55

Newell, E. B. 1970, ApJ, 159, 443

Nguyen, D. D., Seth, A. C., den Brok, M., et al. 2017, ApJ, 836, 237

Noyola, E. 2010, BAAS, 42, 445

Noyola, E., \& Baumgardt, H. 2011, ApJ, 743, 52

Noyola, E., \& Gebhardt, K. 2006, AJ, 132, 447

Noyola, E., Gebhardt, K., \& Bergmann, M. 2008, ApJ, 676, 1008

Nyland, K., Davis, T. A., Nguyen, D. D., et al. 2017, ApJ, 845, 50

O’Leary, R. M., Rasio, F. A., Fregeau, J. M., et al. 2006, ApJ, 637, 937

Ortolani, S., Barbuy, B., Bica, E., et al. 1999, A\&A, 350, 840

Ortolani, S., Barbuy, B., Bica, E., Zoccali, M., \& Renzini, A. 2007, A\&A 470, 1043

Ortolani, S., Bica, E., \& Barbuy, B. 2003, A\&A, 402, 565

Paltrinieri, B., Ferraro, F. R., Paresce, F., \& de Marchi, G. 2001, AJ, 121, 3114

Pavlinsky, M. N., Grebenev, S. A., \& Sunyaev, R. A. 1994, ApJ, 425, 110

Pellegrini, S. 2005, ApJ, 624, 155

Perera, B. B. P., Stappers, B. W., Lyne, A. G., et al. 2017, MNRAS, 468, 2114

Peuten, M., Brockamp, M., Küpper, A. H. W., \& Kroupa, P. 2014, ApJ, 795, 116

Pfahl, E., \& Rappaport, S. 2001, ApJ, 550, 172

Picard, A., \& Johnston, H. M. 1995, A\&AS, 112, 89

Plotkin, R. M., Markoff, S., Kelly, B. C., Körding, E., \& Anderson, S. F. 2012, MNRAS, 419, 267

Pooley, D., \& Rappaport, S. 2006, ApJL, 644, L45

Portegies Zwart, S. F., Baumgardt, H., McMillan, S. L. W., et al. 2006, ApJ, 641,319

Portegies Zwart, S. F., Dewi, J., \& Maccarone, T. 2004, MNRAS, 355, 413

Portegies Zwart, S. F., \& McMillan, S. L. W. 2002, ApJ, 576, 899

Possenti, A., D'Amico, N., Manchester, R. N., et al. 2003, ApJ, 599, 475

Prager, B. J., Ransom, S. M., Freire, P. C. C., et al. 2017, ApJ, 845, 148

Predehl, P., Hasinger, G., \& Verbunt, F. 1991, A\&A, 246, L21

Ransom, S. M., Hessels, J. W. T., Stairs, I. H., et al. 2005, Sci, 307, 892

Rau, U. 2012, Proc. SPIE, 8500, 85000N

Robitaille, T., \& Bressert, E. 2012, APLpy: Astronomical Plotting Library in Python, Astrophysics Source Code Library, ascl:1208.017

Rodriguez, C. L., Chatterjee, S., \& Rasio, F. A. 2016, PhRvD, 93, 084029

Saracino, S., Dalessandro, E., Ferraro, F. R., et al. 2015, ApJ, 806, 152

Sault, R. J., Teuben, P. J., \& Wright, M. C. H. 1995, in Proc. ASP Conf. Ser 77, adass IV, ed. R. A. Shaw, H. E. Payne, \& J. J. E. Hayes (San Francisco, CA: ASP), 433

Shawl, S. J., \& White, R. E. 1986, AJ, 91, 312

Soria, R., Musaeva, A., Wu, K., et al. 2017, MNRAS, 469, 886

Sosin, C., \& King, I. R. 1995, AJ, 109, 639

Spergel, D. N. 1991, Natur, 352, 221

Stacey, W. S., Heinke, C. O., Cohn, H. N., Lugger, P. M., \& Bahramian, A. 2012, ApJ, 751, 62

Strader, J., Chomiuk, L., Maccarone, T. J., et al. 2012, ApJL, 750, L27

Taylor, M. B. 2005, adass XIV, 347, 29 
Trager, S. C., King, I. R., \& Djorgovski, S. 1995, AJ, 109, 218

Trenti, M. 2006, arXiv:astro-ph/0612040

Valenti, E., Ferraro, F. R., \& Origlia, L. 2007, AJ, 133, 1287

Valenti, E., Ferraro, F. R., \& Origlia, L. 2010, MNRAS, 402, 1729

Valenti, E., Origlia, L., \& Ferraro, F. R. 2005, MNRAS, 361, 272

van der Marel, R. P., \& Anderson, J. 2010, ApJ, 710, 1063

van Loon, J. T., Stanimirović, S., Evans, A., \& Muller, E. 2006, MNRAS, 365,1277

Vanbeveren, D., Belkus, H., van Bever, J., \& Mennekens, N. 2009, Ap\&SS, 324,271

Vanderbeke, J., de Propris, R., de Rijcke, S., et al. 2015, MNRAS, 450, 2692

Vesperini, E., McMillan, S. L. W., D'Ercole, A., \& D'Antona, F. 2010, ApJL, 713, L41

Volonteri, M., \& Perna, R. 2005, MNRAS, 358, 913
Watkins, L. L., van der Marel, R. P., Bellini, A., et al. 2016, MmSAI, 87, 610 Watkins, L. L., van der Marel, R. P., Bellini, A., \& Anderson, J. 2015, ApJ, 812,149

Webb, N., Cseh, D., Lenc, E., et al. 2012, Sci, 337, 554

Webb, N. A., Guérou, A., Ciambur, B., et al. 2017, A\&A, 602, A103

Wells, D. C. 1985, in Data Analysis in Astronomy, ed. V. Di Gesù et al. (New York: Plenum Press), 195

Wilson, W. E., Ferris, R. H., Axtens, P., et al. 2011, MNRAS, 416, 832

Wolszczan, A., Kulkarni, S. R., Middleditch, J., et al. 1989, Natur, 337, 531

Woods, T. E., Heger, A., Whalen, D. J., et al. 2017, ApJL, 842, L6

Wrobel, J. M., Greene, J. E., \& Ho, L. C. 2011, AJ, 142, 113

Wrobel, J. M., Miller-Jones, J. C. A., \& Middleton, M. J. 2016, AJ, 152, 22

Wrobel, J. M., Nyland, K. E., \& Miller-Jones, J. C. A. 2015, AJ, 150, 120

Zinn, R., \& Barnes, S. 1998, AJ, 116, 1736 